\title{
Natural dualities for quasivarieties generated by a finite commutative ring
}

\author{
DAvid M. Clark, Pawee M. IDZiak, Lousindi R. SABOurin, Csaba Szabó \\ AND ROSS WILLARD
}

Dedicated to Viktor Aleksandrovich Gorbunov

\begin{abstract}
Let $R$ be a finite commutative ring with identity. If the Jacobson radical of $R$ annihilates itself, then the quasivariety generated by $R$ is dually equivalent to a category of structured Boolean spaces obtained in a natural way from $R$. If on the other hand the radical of $R$ does not annihilate itself, then no such natural dual equivalence is possible. To illustrate the first result, a dual equivalence for the quasivariety generated by the ring $\mathbb{Z}_{p^{2}}$, where $p$ is prime, is given.
\end{abstract}

\section{Introduction}

Stone's 1936 [18] description of Boolean rings has two parts. In modern language it says that every Boolean ring is isomorphic to the ring of all clopen subsets of some Boolean space, and that the association between Boolean rings and the corresponding Boolean spaces is a dual equivalence between the quasivariety of Boolean rings and the category of Boolean spaces. The quasivariety of Boolean rings consists of all rings with identity that are embeddable into a power of the two element field $F_{2}$. In 1968 Arens and Kaplansky [1] extended this result to the quasivariety consisting of all rings with identity that are embeddable into a power of the field $F_{q}$. Taking $G$ to be the automorphism group of $F_{q}$ and $X$ to be a Boolean space continuously acted upon by $G$, they constructed the ring of $G$-stable continuous functions from $X$ into $F_{q}$. Their theorem says that

- every commutative ring with identity in the quasivariety generated by $F_{q}$ is isomorphic to the ring of $G$-stable continuous functions from some Boolean $G$-space $X$ into $F_{q}$, and that

Presented by Professors Kira Adaricheva and Wieslaw Dziobiak.

Received October 1, 2000; accepted in final form December 27, 2000.

2000 Mathematics Subject Classfication: Primary 13Mxx; Secondary 18A40, 08C15.

Key words and phrases: Natural dualities, commutative rings, quasivarieties.

The support of Polish KBN grant 2P03A-031-09 (Idziak), the NSERC of Canada (Willard), and the Fields Institute for Research in Mathematical Sciences (Idziak, Szabó, Willard) are gratefully acknowledged. 
- the association between these rings and the corresponding Boolean $G$-spaces is a dual equivalence between the quasivariety generated by $F_{q}$ and the category of Boolean $G$-spaces.

Since the publication of Arens and Kaplansky's work there has been a steady and growing stream of similar results, compiled and exposited in the text [2] of Clark and Davey, where $F_{q}$ is replaced by an arbitrary finite algebra $\underline{\mathbf{M}}$. Leaving the technical definitions to the next section, we say that $\underline{\mathbf{M}}$ is dualizable if a representation of the former type exists, that $\underline{\mathbf{M}}$ is fully dualizable if a dual equivalence of the latter type exists, and that $\underline{\mathbf{M}}$ is strongly dualizable if it is fully dualizable in a particularly nice way. The literature now abounds with examples of these constructions. (See [2], Chapter 4.) Davey and Willard [10] and Saramago [17] (Chapter 6) proved that a quasivariety which is dualizable with respect to one finite generator is also dualizable with respect to every other finite generator. Applying this fact to the examples given in [2], we find many quasivarieties in which every finite member is dualizable. For example, every finite Boolean algebra, abelian group, semilattice or vector space is dualizable. In contrast Quackenbush and Szabó [16] have shown that every finite nilpotent non-abelian group is non-dualizable.

However, the general problem as to whether or not an arbitrary finite algebra $\underline{\mathbf{M}}$ is or is not (strongly) dualizable remains challenging and difficult, raising the interesting question as to whether or not the problem is even recursively decidable. This situation has led several investigators to restrict the dualizability problem to a particular class $\mathcal{C}$ of algebras, and attempt to determine exactly which members of $\mathcal{C}$ are dualizable. Two notable results describe a class $\mathcal{C}$ that is properly partitioned into dualizable and non-dualizable members. Within congruence-distributive varieties, the dualizability problem has been reduced to the problem of deciding if the algebra in question has a near-unanimity term (Clark, Davey, Heindorf, Krauss, McKenzie, Werner: [9], [5]-if; [8]-only if):

- A finite algebra that generates a congruence-distributive variety is dualizable if and only if it has a near-unanimity term.

Illustrating the difficulty of the dualizability problem, Clark, Davey and Pitkethly [4] give a complete solution for a class of presumably very simple algebras:

- Of the 699 monoids of unary operations on a given three element set, exactly 432 determine a dualizable unary algebra and 267 determine a non-dualizable unary algebra.

The goal of this paper is to give a complete solution to the dualizability problem for finite commutative rings with identity. Interestingly, the third condition of Theorem 1.1 below was shown by Dziobiak [11] to also characterize four other classes of finite rings with identity: those that have a finitely based quasi-equational theory, those whose generated 
quasivariety has only finitely many sub-quasivarieties, those that generate a residually small variety and those that generate a residually finite variety.

THEOREM 1.1. Let $\mathbf{R}$ be a finite commutative ring with identity. Then the following are equivalent:

1. $\mathbf{R}$ is dualizable;

2. $\mathbf{R}$ is strongly dualizable;

3. the Jacobson radical $J(\mathbf{R})$ of $R$ is self annihilating, that is, $a b=0$ whenever $a, b \in J(\mathbf{R})$.

The significance of this result lies in the fact that commutative rings with identity are now the only class $\mathcal{C}$ of finite algebras for which the following three statements can be made.

- $\mathcal{C}$ is a class of algebras of historical interest in classical algebra.

- A finite algorithm is known that will determine whether or not a member of $\mathcal{C}$ is dualizable.

- The dualizable members of $\mathcal{C}$ form a proper and algebraically interesting subclass of $\mathcal{C}$.

\section{Natural dualities}

Before explaining the general context in which we work, let us say a little more about the result of Arens and Kaplansky. Fix a finite field $F$, let $\mathcal{A}$ and $\mathcal{X}$ denote respectively the quasivariety of rings generated by $F$ and the category of Boolean $G$-spaces where $G=\operatorname{Aut}(F)$, and let $\mathbf{F} \in \mathcal{A}$ and $\mathbf{F} \in \mathcal{X}$ denote the two incarnations of $F$ as a ring and as a discrete $G$-space respectively. $\mathcal{A}$ consists of all rings with identity which are isomorphic to subrings of powers of $\underline{\mathbf{F}}$; we denote this fact by $\mathcal{A}=\mathbb{I S P}(\underline{\mathbf{F}})$. In the category of topological spaces with an action one can form powers (using the product topology and the inherited coordinatewise action) and subobjects (on subsets which are closed under the action). Then $\mathcal{X}$ coincides with the class of topological spaces with action which are isomorphic to topologically closed subobjects of powers of $\underset{\sim}{\mathbf{F}} ;$ we denote this fact by $\mathcal{X}=\mathbb{S}_{c} \mathbb{P}^{+}(\underset{\sim}{\mathbf{F}})$.

The "natural" dual equivalence between $\mathcal{A}$ and $\mathcal{X}$ is given by the contravariant homfunctors $D=\operatorname{Hom}(-, \underline{\mathbf{F}})$ and $E=\operatorname{Hom}(-, \mathbf{F})$ and the "evaluation map" natural transformations $e: 1_{\mathcal{A}} \rightarrow E D$ and $\varepsilon: 1_{\mathcal{X}} \rightarrow D E$. More specifically, for $R \in \mathcal{A}, a \in R$ and $h: R \rightarrow \underline{\mathbf{F}}$, we define $e^{R}(a)(h)=h(a)$, and for $X \in \mathcal{X}, x \in X$ and $\alpha: X \rightarrow \underset{\sim}{\mathbf{F}}$, we define $\epsilon^{X}(x)(\alpha)=\alpha(x)$. Here $E(X)$ inherits its structure as a subring of the power $\underline{\mathbf{F}}^{X}$, for any $X \in \mathcal{X}$. Similarly, if $R \in \mathcal{A}$, then $D(R)$ inherits its topology and $G$-action as a closed subobject of ${\underset{\mathbf{F}}{R}}^{R}$. To say that $D, E, e, \varepsilon$ give a dual equivalence amounts to saying that they give a dual adjunction and for every $R \in \mathcal{A}$ and $X \in \mathcal{X}$, the natural embeddings $e^{R}: R \hookrightarrow E D(R)$ and $\varepsilon^{X}: X \hookrightarrow D E(X)$ are surjective.

The previous discussion may be carried out in a quite general setting (see [2], Chapter 1). Briefly, suppose $\underline{\mathbf{M}}$ is any finite algebraic structure (such as a ring, group, or semigroup), let 
$\mathbf{M}$ be a discrete space having the same universe as $\underline{\mathbf{M}}$ and enriched with additional structure $\Sigma$, and let $\mathcal{A}=\mathbb{I S P}(\underline{\mathbf{M}})$ and $\mathcal{X}=\mathbb{S}_{c} \mathbb{P}^{+}(\underline{\mathbf{M}})$. Provided $\Sigma$ is "compatible with $\underline{\mathbf{M}}$ " in a sense to be explained below, the hom-functors $D=\operatorname{Hom}(-, \underline{\mathbf{M}})$ and $E=\operatorname{Hom}(-, \underline{\mathbf{M}})$ and evaluation map natural transformations $e$ and $\varepsilon$ will establish at least a dual adjunction between $\mathcal{A}$ and $\mathcal{X}$. If for every $\mathbf{A} \in \mathcal{A}$ the natural embedding $e^{\mathbf{A}}: \mathbf{A} \hookrightarrow E D(\mathbf{A})$ is surjective, then we say that $\mathcal{A}$ is dualized by $\Sigma$ (or by $\underset{\sim}{\mathbf{M}}$ ). If in addition the natural embedding $\varepsilon^{\mathbf{X}}: \mathbf{X} \hookrightarrow \operatorname{DE}(\mathbf{X})$ is surjective for every $\mathbf{X} \in \mathcal{X}$, then we say that $\mathcal{A}$ is fully dualized by $\Sigma$. In the former case, $\mathcal{A}$ is dually equivalent to a full subcategory of $\mathcal{X}$. In the latter case it is dually equivalent to $\mathcal{X}$ itself.

It is argued in [2, §§1.4 and 2.3] that "additional structure" should mean a collection $\Sigma$ of operations and relations, each having a fixed but otherwise arbitrary arity and defined on $M$ (the common universe of $\underline{\mathbf{M}}$ and $\underset{\sim}{\mathbf{M}})$. The operations in $\Sigma$ need not be total, nor must the arities of the operations and relations be finite. That is, if $\lambda$ is an ordinal, then an acceptable $\lambda$-ary operation is any map $f: U \rightarrow M$ where $\varnothing \neq U \subseteq M^{\lambda}$; and if $\lambda \neq 0$, then an acceptable $\lambda$-ary relation is any $r \subseteq M^{\lambda}$.

By [2, Theorem 1.5.2], the correct notion of "compatibility of $\Sigma$ with $\underline{\mathbf{M}}$ " is the following: each $\lambda$-ary relation in $\Sigma$ must be the universe of a subalgebra of $\underline{\mathbf{M}}^{\lambda}$, and each $\lambda$-ary operation $f \in \Sigma$ must be a homomorphism from a subalgebra of $\underline{\mathbf{M}}^{\lambda}$ to $\underline{\mathbf{M}}$. Operations and relations with these properties are said to be algebraic over $\underline{\mathbf{M}}$. Returning to the example of a finite field $\underline{\mathbf{F}}$, the relevant algebraic operation is the Frobenius automorphism as an operation of arity 1 .

There is a notion, called "strong" duality, which has been known for some time to imply full duality and which receives considerable attention in the book [2]. (See Definition 4.4.) As the reader will see, our proofs of full duality are in fact proofs of strong duality. The point to be made here is that if $\operatorname{ISP}(\underline{\mathbf{M}})$ is dualized (strongly dualized) by $\Sigma$, then it is also dualized (strongly dualized) by any collection of algebraic operations and relations containing $\Sigma$. (At present it is unknown whether 'fully dualized' has the same property; this is one reason to prefer strong duality over full duality.)

It turns out [2, §2.3] that $\mathbb{I S P}(\underline{\mathbf{M}})$ is always strongly dualized by the proper class of all operations and relations of all possible arities (infinite as well as finite) which are algebraic over $\underline{\mathbf{M}}$, but we consider this to be cheating. Roughly speaking, we want to keep the arities of the operations and relations in $\Sigma$ small (preferably finite), hoping that topological closure will suffice to compensate for the absence of the infinitary algebraic operations and relations. If $\kappa$ is a cardinal, we say that $\underline{\mathbf{M}}$ is [fully, strongly] $\kappa$-dualizable if $\mathbb{I S P}(\underline{\mathbf{M}})$ is [fully, strongly] dualized dualized) by the set of all $<\kappa$-ary operations and relations algebraic over $\underline{\mathbf{M}}$. In this terminology, the result of Arens and Kaplansky implies that each finite field is strongly 2 -dualizable. In general, what is desired is that $\underline{\mathbf{M}}$ be strongly $\kappa$-dualizable for some very small cardinal $\kappa$, resulting in a more tractable dual category $\mathcal{X}$. (In particular, the standard notion of dualizable is our $\omega$-dualizable.) We will prove the following strengthened version of Theorem 1.1. 
THEOREM 2.1. Let $\underline{\mathbf{R}}$ be a finite commutative ring with identity. Then the following are equivalent:

1. $\underline{\mathbf{R}}$ is $\kappa$-dualizable for some cardinal $\kappa$;

2. $\underline{\mathbf{R}}$ is strongly 4-dualizable;

3. the Jacobson radical $J(\underline{\mathbf{R}})$ of $\underline{\mathbf{R}}$ is self annihilating, that is, $a b=0$ whenever $a, b \in$ $J(\underline{\mathbf{R}})$.

Even when the Jacobson radical of $\underline{\mathbf{R}}$ is self annihilating, the set $\Sigma$ of algebraic operations and relations of $\underline{\mathbf{R}}$ of arity at most 3 is generally large enough to produce a rather cumbersome dual object $\underset{\sim}{\mathbf{R}}$ and a completely impenetrable dual category $\mathbb{I S}_{c} \mathbb{P}^{+}(\underset{\sim}{\mathbf{R}})$. A natural game to play is to find a smaller, more manageable subset of $\Sigma$ which still does the job. The result of Arens and Kaplansky is one instance; see [2, Theorem 4.2.5] for a similar result in case $\underline{\mathbf{R}}=\mathbb{Z}_{p q}$ where $p, q$ are distinct primes.

In Theorem 7.1 we make the first move of this game in the general case. It is our hope that ring theorists will be able to use this theorem as a starting point to obtain useful dual equivalences for quasivarieties generated by specific finite commutative rings with self-annihilating radicals. In the last section we give an example of what is required by explicitly working out a dual category for the ring $\mathbb{Z}_{p^{2}}$ where $p$ is a prime.

We shall draw freely from the general theory of natural dualities as it is expounded in [2]. Some additions to the theory which we need here, and which have already found application in [10, 15, 13, 19], are placed in Section 4. Lambek's book [14] provides a good reference for the ring theory we use.

\section{Nondualizability}

THEOREM 3.1. Let $\underline{\mathbf{R}}$ be a finite commutative ring (with or without identity) whose radical is not self-annihilating. Then $\underline{\mathbf{R}}$ is not $\kappa$-dualizable for any cardinal $\kappa$.

This section consists of the proof of Theorem 3.1. This theorem is slightly stronger than (1) $\Rightarrow$ (3) of Theorem 2.1 in two ways: first, it applies to commutative rings without an identity element. Second, if the ring $\underline{\mathbf{R}}$ happens to have an identity element, then the statement of the theorem has two possible interpretations, depending on whether or not subrings in $\mathbb{I S P}(\underline{\mathbf{R}})$ are required to contain the identity element of the larger ring and homomorphisms are required to preserve the identity elements of the respective rings (i.e., whether the identity element of $\underline{\mathbf{R}}$ is named by a formal symbol). Our proof establishes $\kappa$-nondualizability under both interpretations.

We begin with three elementary observations from [2] that hold for any finite algebra

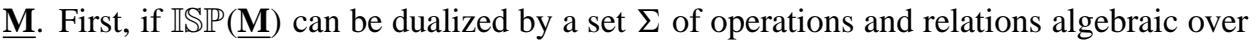


$\underline{\mathbf{M}}$, then it can be dualized by a set consisting of relations only (as in this context operations can be replaced by their graphs [2, Theorem 2.1.2]).

Second, suppose $\Sigma$ is a collection of operations and relations algebraic over $\underline{\mathbf{M}}$, and let the topological structure $\underset{\sim}{\mathbf{M}}$, the category $\mathcal{X}$ and the functors $D, E$ be defined relative to $\Sigma$ as in the introduction. Then for each $\mathbf{A} \in \mathbb{I S P}(\underline{\mathbf{M}})$ the universe of $E D(\mathbf{A})$ has the following concrete characterization. Let $r$ be a $\lambda$-ary relation on $M$. A $\lambda$-tuple $\left(h_{\alpha}\right)_{\alpha<\lambda} \in \operatorname{Hom}(\mathbf{A}, \underline{\mathbf{M}})^{\lambda}$ is coordinatewise in $r$ if $\left(h_{\alpha}(a)\right)_{\alpha<\lambda} \in r$ for all $a \in A$. If $\varphi: \operatorname{Hom}(\mathbf{A}, \underline{\mathbf{M}}) \rightarrow M$ is any function, then we say that $\varphi$ preserves $r$ if for every $\lambda$-tuple $\left(h_{\alpha}\right)_{\alpha<\lambda} \in \operatorname{Hom}(\mathbf{A}, \underline{\mathbf{M}})^{\lambda}$ which is coordinatewise in $r$ we have $\left(\varphi\left(h_{\alpha}\right)\right)_{\alpha<\lambda} \in r$. Now we can state the characterization: the universe of $E D(\mathbf{A})$ consists of those functions $\varphi: \operatorname{Hom}(\mathbf{A}, \underline{\mathbf{M}}) \rightarrow M$ which are continuous, preserve every relation $r \in \Sigma$, and preserve the graph of every operation $f \in \Sigma$. To remind the reader of this fact, we shall occasionally use $C_{\Sigma}(\operatorname{Hom}(\mathbf{A}, \underline{\mathbf{M}}), M)$ to denote the universe of $E D(\mathbf{A})$.

Third, if the structure $\underset{\sim}{\mathbf{M}}$, the category $\mathcal{X}$, the functors $D, E$ and the natural transformations $e, \varepsilon$ are defined relative to $\Sigma$ as in the introduction, then for each $\mathbf{A} \in \mathbb{I S P}(\underline{\mathbf{M}})$ the range of the natural embedding $e^{\mathbf{A}}: \mathbf{A} \hookrightarrow E D(\mathbf{A})$ is always the same: it is the set $\left\{e^{\mathbf{A}}(a): a \in A\right\}$ of the "evaluation-at- $a$ maps" each defined by $e^{\mathbf{A}}(a)(h)=h(a)$.

Thus we shall accomplish our goal by constructing, for each infinite cardinal $\kappa$, a ring $\mathbf{S}_{\kappa} \in \mathbb{I S P}(\underline{\mathbf{R}})$ and a map $\varphi_{\kappa}: \operatorname{Hom}\left(\mathbf{S}_{\kappa}, \underline{\mathbf{R}}\right) \rightarrow R$ which is continuous, preserves every $<\kappa$-ary relation algebraic over $\underline{\mathbf{R}}$, and yet differs from $e^{\mathbf{S}_{\kappa}}(a)$ for every $a \in S_{\kappa}$. The next lemma is our main tool.

LEMMA 3.2. Let $\underline{\mathbf{M}}$ be a finite algebra, $\mathbf{A} \in \mathbb{I S P}(\underline{\mathbf{M}})$, and $\varphi: \operatorname{Hom}(\mathbf{A}, \underline{\mathbf{M}}) \rightarrow M$.

1. $\varphi$ is continuous if and only if there exists a finite set $A_{0} \subseteq A$ such that for all $h, h^{\prime} \in \operatorname{Hom}(\mathbf{A}, \underline{\mathbf{M}})$, if $\left.h\right|_{A_{0}}=\left.h^{\prime}\right|_{A_{0}}$ then $\varphi(h)=\varphi\left(h^{\prime}\right)$.

2. For a nonzero ordinal $\lambda$, $\varphi$ preserves all $\lambda$-ary relations algebraic over $\underline{\mathbf{M}}$ if and only if for every set $H_{0} \subseteq \operatorname{Hom}(\mathbf{A}, \underline{\mathbf{M}})$ with $0<\left|H_{0}\right| \leq|\lambda|$ there exists $a \in A$ such that $\left.\varphi\right|_{H_{0}}=\left.e^{\mathbf{A}}(a)\right|_{H_{0}}$.

Proof. Item 1 is true by a simple compactness argument. To prove item 2 , assume that $\varphi$ preserves all $\lambda$-ary algebraic relations of $\underline{\mathbf{M}}$ and let $\varnothing \neq H_{0} \subseteq \operatorname{Hom}(\mathbf{A}, \underline{\mathbf{M}})$ with $\left|H_{0}\right| \leq|\lambda|$. Enumerate $H_{0}=\left\{h_{\alpha}: \alpha<\lambda\right\}$. For each $a \in A$ define $f_{a} \in M^{\lambda}$ by $f_{a}(\alpha)=e^{\mathbf{A}}(a)\left(h_{\alpha}\right)$ and put $r=\left\{f_{a}: a \in A\right\}$. It can be checked that the map $a \mapsto f_{a}$ is a homomorphism from $\mathbf{A}$ to $\underline{\mathbf{M}}^{\lambda}$ with range $r$; therefore $r$ is the universe of a subalgebra of $\underline{\mathbf{M}}^{\lambda}$ and so is a $\lambda$-ary relation algebraic over $\underline{\mathbf{M}}$.

Thus $\varphi$ preserves $r$. Now consider the $\lambda$-tuple $\tau=\left(h_{\alpha}\right)_{\alpha<\lambda} \in \operatorname{Hom}(\mathbf{A}, \underline{\mathbf{M}})^{\lambda}$. Clearly $\tau$ is coordinatewise in $r$. As $\varphi$ preserves $r$, we must have $\left(\varphi\left(h_{\alpha}\right)\right)_{\alpha<\lambda}=f_{a} \in r$ for some $a \in A$, which proves the "only if" direction of item 2 . The converse is immediate. 


\subsection{The easy case}

In this subsection we prove Theorem 3.1 in the special case that $\underline{\mathbf{R}} \models x^{2}=y^{2}=0 \rightarrow$ $x y=0$. This case includes, e.g., the rings $\mathbb{Z}_{p^{3}}$ ( $p$ a prime). This subsection is redundant in the sense that the next subsection gives a general argument covering all cases. However, we find it easier to introduce (and believe it will be easier to read) some of the ideas in the context of this special case.

CLAIM 3.3. There exist $k \in\{2,3\}$ and $a, b \in R$ such that

1. $\underline{\mathbf{R}} \models x^{2}=y^{k}=0 \rightarrow x y=0$.

2. $\overline{a^{k}}=a^{2} b=a b^{2}=0$.

3. $a b \neq 0$.

Proof. Let $J$ be the radical of $\underline{\mathbf{R}}$. Choose $n \geq 1$ maximal such that $J^{2 n} \neq 0$, and let $I=J^{n}$. Thus $I^{2} \neq 0$ while $I^{4}=0$. In particular, $I=x^{4}=0$.

Assume first that $\underline{\mathbf{R}}=x^{2}=y^{4}=0 \rightarrow x y=0$. Then $\underline{\mathbf{R}} \models y^{4}=0 \rightarrow y^{3}=0$. Choose $a, c \in I$ with $a c \neq 0$. Because $a \in I$ we get $a^{4}=0$ and therefore $a^{3}=0$. Similarly, $c^{4}=0$. Since $a c \neq 0$ we must have $a^{2} \neq 0$ (else $a, c$ would violate $\underline{\mathbf{R}} \models x^{2}=$ $\left.y^{4}=0 \rightarrow x y=0\right)$. Then $a, b:=a$ and $k:=3$ witness the required conditions.

Assume on the other hand that there exist $a, b \in R$ such that $a^{2}=b^{4}=0$ while $a b \neq 0$. Let $c=b^{2}$; then $a^{2}=c^{2}=0$, so by the hypothesis of this subsection we have $a c=0$, i.e., $a b^{2}=0$. Thus $a, b$ and $k:=2$ witness the required conditions.

Let $k, a, b$ be fixed as in the previous claim and put $c:=a b \neq 0$. We define some elements of the ring $\underline{\mathbf{R}}^{4}$ as follows: $R$.

$$
\underline{\mathbf{a}}=[a, a, 0,0], \quad \underline{\mathbf{b}}=[b, 0, b, 0], \quad \underline{\mathbf{c}}=[c, 0,0,0], \quad \hat{r}=[r, r, r, r],
$$

where $r$ ranges over $R$. Also define a map $v: \underline{\mathbf{R}}^{4} \rightarrow \underline{\mathbf{R}}$ by

$$
v([w, x, y, z])=w-x-y+z .
$$

The facts we need to know about the above elements and the map $v$ are:

1. $\underline{\mathbf{a}}^{k}=\underline{\mathbf{a}}^{2} \underline{\mathbf{b}}=\underline{\mathbf{b}}^{2} \underline{\mathbf{a}}=\hat{0}$, while $\underline{\mathbf{a b}}=\underline{\mathbf{c}}$.

2. $v\left(\underline{\mathbf{a}}^{n}\right)=v\left(\underline{\mathbf{b}}^{n}\right)=v(\hat{r})=0$ for all $n \geq 1$ and $r \in R$, while $v(\underline{\mathbf{c}}) \neq 0$.

3. $v$ is $\mathbf{R}$-linear.

Fix an infinite cardinal $\kappa$. We define elements $\alpha, \theta_{j}(j<\kappa)$ and $\eta_{r}(r \in R)$ of $\left(\underline{\mathbf{R}}^{4}\right)^{\kappa}$ as follows.

$$
\begin{aligned}
& \alpha(i)=\underline{\mathbf{a}} \quad \text { for all } i<\kappa, \\
& \theta_{j}(i)= \begin{cases}\frac{\mathbf{b}}{\hat{\hat{0}}} & \text { if } i=j \\
0 & \text { otherwise }\end{cases} \\
& \eta_{r}(i)=\hat{r} \quad \text { for all } i<\kappa \text {. }
\end{aligned}
$$


Also define $\beta_{j}=\theta_{0}-\theta_{j}$ and $\gamma_{j}=\alpha \beta_{j}$ for $j<\kappa$, and $\gamma=\alpha \theta_{0}$. Thus $\beta_{0}=\gamma_{0}=0$ and for $0<j<\kappa$,

$$
\begin{aligned}
& \alpha=(\underline{\mathbf{a}}, \underline{\mathbf{a}}, \underline{\mathbf{a}}, \ldots, \underline{\mathbf{a}}, \underline{\mathbf{a}}, \underline{\mathbf{a}}, \ldots), \\
& \beta_{j}=(\underline{\mathbf{b}}, \hat{0}, \hat{0}, \ldots, \hat{0},-\underline{\mathbf{b}}, \hat{0}, \ldots) \text {, } \\
& \gamma_{j}=(\underline{\mathbf{c}}, \hat{0}, \hat{0}, \ldots, \hat{0},-\underline{\mathbf{c}}, \hat{0}, \ldots) \text {, } \\
& \uparrow \\
& j \\
& \eta_{r}=(\hat{r}, \hat{r}, \hat{r}, \ldots, \hat{r}, \hat{r}, \hat{r}, \ldots) \text {, }
\end{aligned}
$$

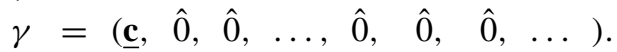

Let $\mathbf{S}_{\kappa}$ be the subring of $\left(\underline{\mathbf{R}}^{4}\right)^{\kappa}$ generated by the set

$\{\alpha\} \cup\left\{\beta_{j}: j<\kappa\right\} \cup\left\{\eta_{r}: r \in R\right\}$.

CLAIM 3.4. (Parity check) For every $\delta \in S_{\kappa}$ there exists a finite subset $D \subseteq \kappa$ such that for all finite sets $F$ with $D \subseteq F \subseteq \kappa$,

$$
\sum_{i \in F} v(\delta(i))=0
$$

Hence $\gamma \notin S_{K}$.

Proof. Let $M$ be the set of all $\delta \in\left(R^{4}\right)^{\kappa}$ satisfying the above condition. Then $M$ is an $\underline{\mathbf{R}}$-submodule of $\left(\underline{\mathbf{R}}^{4}\right)^{\kappa}$. Let $G=\{\alpha\} \cup\left\{\beta_{j}: 0<j<\kappa\right\}$ and let $H$ be the closure of $G$ under multiplication. Then $S_{\kappa}$ is the $\underline{\mathbf{R}}$-submodule of $\left(\underline{\mathbf{R}}^{4}\right)^{\kappa}$ generated by $H \cup\left\{\eta_{r}: r \in R\right\}$, so it suffices to show that $H \cup\left\{\eta_{r}: r \in R\right\} \subseteq M$. We leave this verification to the reader.

Before defining our map $\varphi_{\kappa}: \operatorname{Hom}\left(\mathbf{S}_{\kappa}, \underline{\mathbf{R}}\right) \rightarrow R$, we shall study the restriction of each $h \in \operatorname{Hom}\left(\mathbf{S}_{\kappa}, \underline{\mathbf{R}}\right)$ to the set $\left\{\gamma_{j}: j<\kappa\right\}$. Fix $h \in \operatorname{Hom}\left(\mathbf{S}_{\kappa}, \underline{\mathbf{R}}\right)$ and color the elements of $\kappa$ by assigning to each $j<\kappa$ the value of $h\left(\gamma_{j}\right)$.

CLAIM 3.5. One of the color-classes is cofinite. The union of the finite color-classes has cardinality $<|R|$.

Proof. For $j, \ell<\kappa$ define

$$
j \equiv_{0} \ell \text { iff } h\left(\beta_{j}\right)=h\left(\beta_{\ell}\right) .
$$

Clearly each color-class is a union of $\equiv_{0}$-classes, and there are at most $|R|$ distinct $\equiv_{0}$ classes. It suffices to show that if $j, \ell, m, n$ are distinct members of $\kappa$ and $j \equiv_{0} \ell$ and $m \equiv_{0} n$, then $j, \ell, m, n$ belong to the same color-class. Certainly $j$ and $\ell$ belong to the 
same color-class, as do $m$ and $n$. Furthermore, $h\left(\beta_{j}-\beta_{m}\right)=h\left(\beta_{\ell}-\beta_{n}\right)=: x$. Now in $\left(\underline{\mathbf{R}}^{4}\right)^{\kappa},\left(\beta_{j}-\beta_{m}\right)\left(\beta_{\ell}-\beta_{n}\right)=\left(\theta_{m}-\theta_{j}\right)\left(\theta_{n}-\theta_{\ell}\right)=0$ (since $\theta_{p} \theta_{q}=0$ whenever $\left.p \neq q\right)$. Thus $x^{2}=0$. By Claim 3.3, $x y=0$ for all $y \in R$ such that $y^{k}=0$ (where $k$ is the integer specified in Claim 3.3). Since $\alpha^{k}=0$ in $\left(\underline{\mathbf{R}}^{4}\right)^{\kappa}$ we get $h(\alpha)^{k}=0$ in $\underline{\mathbf{R}}$ and hence $0=x h(\alpha)=h\left(\beta_{j}-\beta_{m}\right) h(\alpha)=h\left(\gamma_{j}\right)-h\left(\gamma_{m}\right)$, proving that $j$ and $m$ belong to the same color-class.

Now define $\varphi_{\kappa}: \operatorname{Hom}\left(\mathbf{S}_{\kappa}, \underline{\mathbf{R}}\right) \rightarrow R$ by letting $\varphi_{\kappa}(h)$ be the color of the cofinite colorclass determined by $h$. We must show three things: (1) that $\varphi_{\kappa}$ is continuous, (2) that $\varphi_{\kappa}$ preserves every $<\kappa$-ary relation algebraic over $\underline{\mathbf{R}}$, and (3) that $\varphi_{\kappa}$ differs from every evaluation-at- $\delta$ map $e^{\mathbf{S}_{\kappa}}(\delta), \delta \in S_{\kappa}$.

(1) Let $S_{0}=\left\{\gamma_{j}: j<2|R|\right\}$. For every $h \in \operatorname{Hom}\left(\mathbf{S}_{\kappa}, \underline{\mathbf{R}}\right)$, the value of $\varphi_{\kappa}(h)$ can be determined from the restriction of $h$ to $S_{0}$, by Claim 3.5. Thus $\varphi_{\kappa}$ is continuous by Lemma 3.2(1).

(2) Suppose $H_{0} \subseteq \operatorname{Hom}\left(\mathbf{S}_{\kappa}, \underline{\mathbf{R}}\right)$ with $\left|H_{0}\right|<\kappa$. For each $h \in H_{0}$ let $C_{h} \subseteq \kappa$ denote the cofinite color-class determined by $h$. By simple cardinality considerations, $\left|\bigcap_{h \in H_{0}} C_{h}\right|=\kappa$, so we can pick $j \in \bigcap_{h \in H_{0}} C_{h}$. Then $\varphi_{\kappa}(h)=h\left(\gamma_{j}\right)$ for every $h \in H_{0}$. By Claim 3.2(2), $\varphi_{\kappa}$ preserves every $<\kappa$-ary relation algebraic over $\underline{\mathbf{R}}$.

(3) For each $i<\kappa$ define the projection maps $\pi_{1}^{i}, \pi_{2}^{i}, \pi_{3}^{i}, \pi_{4}^{i} \in \operatorname{Hom}\left(\mathbf{S}_{\kappa}, \underline{\mathbf{R}}\right)$ so that for all $\delta \in S_{\kappa}$ and all $i<\kappa$,

$$
\delta(i)=\left[\pi_{1}^{i}(\delta), \pi_{2}^{i}(\delta), \pi_{3}^{i}(\delta), \pi_{4}^{i}(\delta)\right] .
$$

An easy computation shows $\varphi_{\kappa}\left(\pi_{1}^{0}\right)=c$ while $\varphi_{\kappa}\left(\pi_{j}^{i}\right)=0$ in all other cases. Now suppose there exists $\delta \in S_{\kappa}$ such that $\varphi_{\kappa}(h)=h(\delta)$ for all $h \in \operatorname{Hom}\left(\mathbf{S}_{\kappa}, \underline{\mathbf{R}}\right)$. In particular, $\pi_{1}^{0}(\delta)=c$ while $\pi_{j}^{i}(\delta)=0$ for all $(i, j) \neq(0,1)$. This forces $\delta=\gamma$; but $\gamma \notin S_{\kappa}$ by Claim 3.4. Thus $\varphi_{\kappa}$ is not the evaluation-at- $\delta$ map for any $\delta \in S_{\kappa}$, which completes the proof that $\underline{\mathbf{R}}$ is not $\kappa$-dualizable.

\subsection{The general case}

Let $\underline{\mathbf{R}}$ be a finite commutative ring whose radical is not self-annihilating. Choose $a, b \in R$ satisfying $a^{2} b=a b^{2}=0$ while $c:=a b \neq 0$. (If $\underline{\mathbf{R}} \models x^{2}=y^{2}=0 \rightarrow x y=0$ then use Claim 3.3; otherwise, use a failure of this implication.) Our construction is an adaptation to several dimensions of an argument developed for certain nilpotent groups by R. Quackenbush and the fourth author [16]. Define $\underline{\mathbf{a}}, \underline{\mathbf{b}}, \underline{\mathbf{c}}, \hat{r} \in R^{4}(r \in R)$ and $v: \underline{\mathbf{R}}^{4} \rightarrow \underline{\mathbf{R}}$ exactly as in the easy case. We have

1. $\underline{\mathbf{a}}^{2} \underline{\mathbf{b}}=\underline{\mathbf{a b}}^{2}=\hat{0}$ while $\underline{\mathbf{a b}}=\underline{\mathbf{c}}$.

2. $v\left(\underline{\mathbf{a}}^{n}\right)=v\left(\underline{\mathbf{b}}^{n}\right)=v(\hat{r})=0$ for all $r \in R$ and $n \geq 1$, while $v(\underline{\mathbf{c}}) \neq 0$.

3. $v$ is $\underline{\mathbf{R}}$-linear. 
Fix an infinite cardinal $\kappa$ and let $G_{\kappa}=\left\{x \in \mathbb{Z}^{\kappa}:\{i<\kappa: x(i) \neq 0\}\right.$ is finite $\}$. We consider $G_{\kappa}$ as a free abelian group and define the standard free generators $e_{i}(i<\kappa)$ by

$$
e_{i}(j)= \begin{cases}1 & \text { if } j=i \\ 0 & \text { otherwise. }\end{cases}
$$

Now define elements $\beta_{x}, \gamma_{x}\left(x \in G_{\kappa}\right), \theta_{x, i}\left(x \in G_{\kappa}\right.$ and $\left.i<\kappa\right), \eta_{r}(r \in R)$, and $\gamma$ of $\left(\underline{\mathbf{R}}^{4}\right)^{G_{\kappa}}$ as follows.

$$
\begin{aligned}
& \beta_{x}(y)=\left\{\begin{aligned}
\underline{\mathbf{a}} & \text { if } y=x \\
-\underline{\mathbf{b}} & \text { if } y=x+e_{i} \text { for some } i<\kappa \\
\underline{\mathbf{b}} & \text { if } y=x-e_{i} \text { for some } i<\kappa \\
\hat{0} & \text { otherwise }
\end{aligned}\right. \\
& \gamma_{x}(y)=\left\{\begin{aligned}
\underline{\mathbf{c}} & \text { if } y=0 \text { and } x \neq 0 \\
-\underline{\mathbf{c}} & \text { if } y=x \text { and } x \neq 0 \\
\hat{0} & \text { otherwise }
\end{aligned}\right. \\
& \theta_{x, i}(y)=\left\{\begin{aligned}
\underline{\mathbf{c}} & \text { if } y=x \\
-\underline{\mathbf{c}} & \text { if } y=x+e_{i} \\
\hat{0} & \text { otherwise }
\end{aligned}\right. \\
& \gamma(y)= \begin{cases}\frac{\mathbf{c}}{\hat{\hat{t}}} & \text { if } y=0 \\
0 & \text { otherwise }\end{cases} \\
& \eta_{r}(y)=\hat{r} \quad \text { for all } y \in G_{\kappa} .
\end{aligned}
$$

Note that $\theta_{x, i}=\beta_{x} \beta_{x+e_{i}}$ for each $x \in G_{\kappa}$ and $i<\kappa$, and that $\left\{\gamma_{x}: x \in G_{\kappa}\right\}$ is contained in the additive subgroup of $\left(\underline{\mathbf{R}}^{4}\right)^{G_{\kappa}}$ generated by $\left\{\theta_{x, i}: x \in G_{\kappa}, i<\kappa\right\}$. Also note that $\beta_{x} \beta_{y} \neq 0$ only if $y=x+n e_{i}$ for some $i<\kappa$ and $n \in\{-2,-1,0,1,2\}$, and that for all $x_{1}, x_{2}, \ldots, x_{n} \in G_{\kappa}, v\left(\beta_{x_{1}} \beta_{x_{2}} \cdots \beta_{x_{n}}(y)\right) \neq 0$ for some $y \in G_{\kappa}$ if and only if $n=2$ and $\left\{x_{1}, x_{2}\right\}=\left\{x, x+e_{i}\right\}$ for some $x \in G_{\kappa}$ and $i<\kappa$.

Let $\mathbf{S}_{\kappa}$ be the subring of $\left(\underline{\mathbf{R}}^{4}\right)^{G_{\kappa}}$ generated by the set

$$
\left\{\beta_{x}: x \in G_{\kappa}\right\} \cup\left\{\eta_{r}: r \in R\right\},
$$

and note that $\left\{\gamma_{x}: x \in G_{\kappa}\right\} \subseteq S_{\kappa}$. The next claim is proved identically to Claim 3.4.

CLAIM 3.6. (Parity check) For every $\delta \in S_{\kappa}$ there exists a finite subset $D \subseteq G_{\kappa}$ such that for all finite sets $F$ with $D \subseteq F \subseteq G_{\kappa}$,

$$
\sum_{x \in F} v(\delta(x))=0 .
$$

Hence $\gamma \notin S_{\kappa}$. 
As in the easy case, we now study the restriction of each $h \in \operatorname{Hom}\left(\mathbf{S}_{\kappa}, \underline{\mathbf{R}}\right)$ to the set $\left\{\gamma_{x}: x \in G_{\kappa}\right\}$. Fix $h \in \operatorname{Hom}\left(\mathbf{S}_{\kappa}, \underline{\mathbf{R}}\right)$ and color the elements of $G_{\kappa}$ by assigning to each $x \in G_{\kappa}$ the value of $h\left(\gamma_{x}\right)$.

CLAIM 3.7. For $i<\kappa$ define $B_{i}=\left\{x \in G_{\kappa}: x\right.$ and $x+e_{i}$ have different colors $\}$. Then $\left|B_{i}\right| \leq 4|R|^{2}$ for each $i<\kappa$.

Proof. We first show that if $C \subseteq B_{i}$ is any set having the property that $x \pm e_{i} \neq y \pm e_{j}$ for all $j<\kappa$ whenever $x, y \in C$ with $x \neq y$, then $|C| \leq|R|$. For suppose $C$ is such a set. If $x \in C$, then as $h\left(\gamma_{x}\right) \neq h\left(\gamma_{x+e_{i}}\right)$ and $\gamma_{x+e_{i}}-\gamma_{x}=\theta_{x, i}=\beta_{x} \beta_{x+e_{i}}$ we have $h\left(\beta_{x}\right) h\left(\beta_{x+e_{i}}\right) \neq 0$. On the other hand, if $x, y \in C$ with $x \neq y$, then by assumption, $\beta_{y} \beta_{x+e_{i}}=0$ and hence $h\left(\beta_{y}\right) h\left(\beta_{x+e_{i}}\right)=0$. This proves $h\left(\beta_{x}\right) \neq h\left(\beta_{y}\right)$ for all $x, y \in C$ with $x \neq y$, and hence $|C| \leq|R|$.

Now suppose $\left|B_{i}\right|>4|R|^{2}$. For each $n \in \mathbb{Z}$ let $B_{i}(n)=\left\{x \in B_{i}: x(i)=n\right\}$ and put $T=\left\{n \in \mathbb{Z}: B_{i}(n) \neq \varnothing\right\}$. Note that for each $n \in T$ the set $B_{i}(n)$ satisfies the condition of the previous paragraph; hence $\left|B_{i}(n)\right| \leq|R|$ for all $n \in T$. Consequently, $|T|>4|R|$. Hence it is possible to choose $T_{0} \subseteq T$ with $\left|T_{0}\right|>|R|$ and $|n-m| \geq 4$ for all $n, m \in T_{0}$ with $n \neq m$. Now choose one element $x_{n}$ from $B_{i}(n)$ for each $n \in T_{0}$ and put $C=\left\{x_{n}: n \in T_{0}\right\}$; the result is a set satisfying the condition of the previous paragraph and such that $|C|>|R|$, a contradiction.

\section{CLAIM 3.8. One of the color-classes is cofinite.}

Proof. Define $W$ to be the subgroup of $G_{\kappa}$ generated by $\left\{e_{i}: 0<i<\kappa\right\}$, and let $f: G_{\kappa} \rightarrow W$ be the projection defined by $f\left(n e_{0}+x\right)=x$ for all $x \in W$ and $n \in \mathbb{Z}$. For each $x \in W$ let $L_{x}=f^{-1}(x)=\left\{x+n e_{0}: n \in \mathbb{Z}\right\}$. Also let $F=f\left(B_{0}\right)$ where $B_{0}$ is defined in Claim 3.7. Thus $F$ is a finite subset of $W$, and if $x \in W \backslash F$ then the "line" $L_{x}$ belongs to a single color-class. Choose $N<\omega$ large enough so that $x \in B_{0}$ implies $|x(0)|<N$, and for each $x \in F$ define $L_{x}^{+}=\left\{x+n e_{0}: n \geq N\right\}$ and $L_{x}^{-}=\left\{x+n e_{0}: n \leq-N\right\}$. Thus $L_{x}^{+}$ and $L_{x}^{-}$are "half-lines" each of which belongs to a single color-class.

Since $F$ is finite and $\kappa \geq 2$ we can choose and fix some $x \in W \backslash F$. We first show that if $y \in F$, then $L_{x}$ and $L_{y}^{+}$belong to the same color-class. Indeed, let $U=\{0<i<\kappa$ : $x(i) \neq y(i)\}$. Note that $U$ is a finite set. Hence $\bigcup\left\{B_{i}: i \in U\right\}$ is a finite set by Claim 3.7. Choose $N_{y}<\omega$ large enough so that $N_{y} \geq N$ and if $z \in \bigcup\left\{B_{i}: i \in U\right\}$ then $|z(0)|<N_{y}$. Then choose $i_{0}, i_{1}, \ldots, i_{n-1} \in U$ (not necessarily distinct) and $\sigma_{0}, \sigma_{1}, \ldots, \sigma_{n-1} \in\{0,1\}$ so that

$$
y=x+\sum_{k=0}^{n-1}(-1)^{\sigma_{k}} e_{i_{k}}
$$

and for $0 \leq j \leq n$ define $x_{j}=x+N_{y} e_{0}+\sum_{k<j}(-1)^{\sigma_{k}} e_{i_{k}}$. Thus $x_{0} \in L_{x}, x_{n} \in L_{y}^{+}$, and for each $j<n$ we have $x_{j+1}-x_{j}= \pm e_{i}$ for some $i \in U$. By the choice of $N_{y}, x_{j}$ and 
$x_{j+1}$ belong to the same color-class for each $j<n$. Hence $x_{0}$ and $x_{n}$ belong to the same color-class, which proves that $L_{x}$ and $L_{y}^{+}$belong to the same color-class.

Similar arguments show that $L_{x}$ and $L_{y}^{-}$belong to the same color-class whenever $y \in F$, and that $L_{x}$ and $L_{y}$ belong to the same color-class whenever $y \in W \backslash F y \neq x$. Thus the color-class containing $L_{x}$ is cofinite.

CLAIM 3.9. More than half of the elements of $\left\{n e_{0}: 0 \leq n \leq 4|R|^{2}\right\}$ belong to the cofinite color-class.

Proof. Let $D=\left\{0 \leq n \leq 4|R|^{2}: n e_{0}\right.$ does not belong to the cofinite color-class $\}$. If $n \in D$, then there must exist $i_{n}<0$ such that $n e_{0}+i_{n} e_{1}$ and $n e_{0}+\left(i_{n}+1\right) e_{1}$ have different colors; similarly, there must exist $j_{n} \geq 0$ such that $n e_{0}+j_{n} e_{1}$ and $n e_{0}+\left(j_{n}+1\right) e_{1}$ have different colors. Thus $n e_{0}+i_{n} e_{1} \in B_{1}$ and $n e_{0}+j_{n} e_{1} \in B_{1}$ for each $n \in D$. Since $\left|B_{1}\right| \leq 4|R|^{2}$ by Claim 3.7 we get $|D| \leq 2|R|^{2}$, which proves the claim.

Now define $\varphi_{\kappa}: \operatorname{Hom}\left(\mathbf{S}_{\kappa}, \underline{\mathbf{R}}\right) \rightarrow R$ by letting $\varphi_{\kappa}(h)$ be the color of the cofinite colorclass determined by $h$. Arguing as in the easy case, $\varphi_{\kappa}$ is continuous by Claim 3.9, preserves all $<\kappa$-ary relations algebraic over $\underline{\mathbf{R}}$ by Claim 3.8, and is not the evaluation-at- $\delta$ map for any $\delta \in S_{\kappa}$ because $\gamma \notin S_{\kappa}$. Hence $\underline{\mathbf{R}}$ is not $\kappa$-dualizable.

\section{Contributions to the general theory}

In this section we give two new tools for proving that a set $\Sigma$ of finitary operations and relations dualizes (or fully dualizes) $\mathbb{I S P}(\underline{\mathbf{M}})$ where $\underline{\mathbf{M}}$ is a finite algebra. These tools are stated in Theorem 4.3 and Theorem 4.11. The tools will be used in Sections 6 and 7; their flavor is to reduce the analysis from $\mathbb{I S P}(\underline{\mathbf{M}})$ to the class $\mathbb{S S P}_{\text {fin }}(\underline{\mathbf{M}})$ of finite members of $\mathbb{I S P}(\underline{M})$.

The following lemma may be found in [12] (Theorem 1, p. 132), or see [2], 1.3.3, for a short proof.

LEMMA 4.1. Suppose that $P$ is a poset in which any two elements have an upper bound, and $F$ is a function with domain $P$ such that for each $x \in P, F(x)$ is a finite nonempty set. Suppose moreover that for all pairs $(x, y) \in P^{2}$ with $x \leq y$ we have a specified function $f_{x, y}: F(y) \rightarrow F(x)$ and that these functions satisf (i) $f_{x, y} \circ f_{y, z}=f_{x, z}$ whenever $x \leq y \leq z$ in $P$, and (ii) $f_{x, x}=\operatorname{id}_{F(x)}$ for all $x \in P$. Then there is a function $\varphi$ with domain $P$ such that $\varphi(x) \in F(x)$ for all $x \in P$, and $f_{x, y}(\varphi(y))=\varphi(x)$ for all $x \leq y$.

Here is a simple application.

LEMMA 4.2. Let $\mathbf{A}$ be a locally finite algebra, $\mathbf{B}$ a finite algebra of the same type, $r$ an $n$-ary relation on $B(1 \leq n<\omega), S$ a finite subset of $A$, and $g_{1}, \ldots, g_{n} \in B^{S}$. 
Suppose that for every finite subalgebra $\mathbf{A}_{1}$ of $\mathbf{A}$ satisfying $S \subseteq A_{1}$ there exist $h_{1}, \ldots, h_{n} \in \operatorname{Hom}\left(\mathbf{A}_{1}, \mathbf{B}\right)$ such that (a) $\left.h_{i}\right|_{S}=g_{i}$ for $i=1, \ldots, n$, and (b) $\left\langle h_{1}(x), \ldots\right.$, $\left.h_{n}(x)\right\rangle \in r$ for all $x \in A_{1}$. Then there exist $h_{1}, \ldots, h_{n} \in \operatorname{Hom}(\mathbf{A}, \mathbf{B})$ such that the same two conditions hold with $\mathbf{A}$ in place of $\mathbf{A}_{1}$.

Proof. Let $P$ be the poset of finite subalgebras of $\mathbf{A}$ which contain $S$, ordered by inclusion. For each $\mathbf{A}_{1} \in P$ let $F\left(\mathbf{A}_{1}\right)$ be the set of all $n$-tuples $\left\langle h_{1}, \ldots, h_{n}\right\rangle \in \operatorname{Hom}\left(\mathbf{A}_{1}, \mathbf{B}\right)^{n}$ which satisfy the conditions (a) and (b) above. Thus $F\left(\mathbf{A}_{1}\right)$ is finite and nonempty for every $\mathbf{A}_{1} \in P$. If $\mathbf{A}_{1} \leq \mathbf{A}_{2}$ in $P$ then define $f_{\mathbf{A}_{1}, \mathbf{A}_{2}}: F\left(\mathbf{A}_{2}\right) \rightarrow F\left(\mathbf{A}_{1}\right)$ by $f_{\mathbf{A}_{1}, \mathbf{A}_{2}}\left(\left\langle h_{1}, \ldots, h_{n}\right\rangle\right)=$ $\left\langle\left. h_{1}\right|_{A_{1}}, \ldots,\left.h_{n}\right|_{A_{1}}\right\rangle$. By Lemma 4.1 there exists a function $\varphi$ which assigns to each $\mathbf{A}_{1} \in P$ an $n$-tuple $\varphi\left(\mathbf{A}_{1}\right)=\left\langle\varphi_{1}\left(\mathbf{a}_{1}\right), \ldots, \varphi_{n}\left(\mathbf{A}_{1}\right)\right\rangle \in F\left(\mathbf{A}_{1}\right)$ such that $\left.\varphi_{i}\left(\mathbf{A}_{2}\right)\right|_{A_{1}}=\varphi_{i}\left(\mathbf{A}_{1}\right)$ for all $i=1, \ldots, n$ whenever $\mathbf{A}_{1} \leq \mathbf{A}_{2}$ in $P$. Define $h_{1}, \ldots, h_{n} \in B^{A}$ so that $\left.h_{i}\right|_{A_{1}}=\varphi_{i}\left(\mathbf{A}_{1}\right)$ for all $i=1, \ldots, n$ and all $\mathbf{A}_{1} \in P$. This works.

Let $\underline{\mathbf{M}}$ be a finite algebra and $\Sigma$ a set of finitary operations and relations algebraic over $\underline{\mathbf{M}}$, and recall the discussion in Section 2. For $\mathcal{K} \subseteq \mathbb{I S P}(\underline{\mathbf{M}})$ we say that $\Sigma$ dualizes $\mathcal{K}$ if $e^{\mathbf{A}}$ is surjective for every $\mathbf{A} \in \mathcal{K}$. The next theorem was discovered by L. Zádori and independently by the fifth author in 1995 (Zádori actually proved something stronger). Zádori's proof has appeared in [20]; we include our proof since it is significantly shorter.

THEOREM 4.3. Suppose $\underline{\mathbf{M}}$ is a finite algebra and $\Sigma$ is a set of finitary algebraic operations and relations of $\underline{\mathbf{M}}$. If $\Sigma$ dualizes $\mathbb{S S P}_{\text {fin }}(\underline{\mathbf{M}})$ and $\Sigma$ is finite, then $\Sigma$ dualizes $\mathbb{I S P}(\underline{\mathbf{M}})$.

Proof. By replacing operations with their graphs, we may assume that $\Sigma$ consists of relations only [2, Theorem 2.1.2]. Let $\mathbf{A} \in \mathbb{I S P}(\underline{\mathbf{M}})$ and $\varphi \in C_{\Sigma}(\operatorname{Hom}(\mathbf{A}, \underline{\mathbf{M}}), M)$. Choose a finite set $A_{0} \subseteq A$ such that for all $h, h^{\prime} \in \operatorname{Hom}(\mathbf{A}, \underline{\mathbf{M}})$, if $\left.h\right|_{A_{0}}=\left.h^{\prime}\right|_{A_{0}}$ then $\varphi(h)=\varphi\left(h^{\prime}\right)$ (see Lemma 3.2(1)). As $\mathbf{A}$ is locally finite, we may assume that $A_{0}$ is the universe of a subalgebra of $\mathbf{A}$. Let $U$ be the set of all pairs $\left(r,\left\langle g_{1}, \ldots, g_{n}\right\rangle\right)$ where $r \in \Sigma$, where $n$ is the arity of $r$, and $g_{1}, \ldots, g_{n} \in \operatorname{Hom}\left(\mathbf{A}_{0}, \underline{\mathbf{M}}\right)$. For each $(r, \bar{g}) \in U$ choose a finite subalgebra $\mathbf{A}_{(r, \bar{g})}$ of $\mathbf{A}$ so that the following is true: if there do not exist $h_{i} \in \operatorname{Hom}(\mathbf{A}, \underline{\mathbf{M}})$ extending the $g_{i}$ and such that $\bar{h}(x) \in r$ for all $x \in A$, then there do not exist $h_{i} \in \operatorname{Hom}\left(\mathbf{A}_{(r, \bar{g})}, \underline{\mathbf{M}}\right)$ extending the $g_{i}$ and such that $\bar{h}(x) \in r$ for all $x \in A_{(r, \bar{g})}$. (This is possible because of Lemma 4.2.) As $U$ is finite, there exists a finite subalgebra $\mathbf{A}_{1}$ of $\mathbf{A}$ whose universe contains $A_{0}$ and $A_{(r, \bar{g})}$ for every $(r, \bar{g}) \in U$.

Fix some $a_{0} \in M$ and define $\varphi^{*}: \operatorname{Hom}\left(\mathbf{A}_{1}, \underline{\mathbf{M}}\right) \rightarrow M$ by

$$
\varphi^{*}(h)= \begin{cases}\varphi\left(h^{\prime}\right) & \text { if there exists } h^{\prime} \in \operatorname{Hom}(\mathbf{A}, \underline{\mathbf{M}}) \text { with }\left.h^{\prime}\right|_{A_{0}}=\left.h\right|_{A_{0}} \\ a_{0} & \text { otherwise. }\end{cases}
$$

$\varphi^{*}$ is well-defined by choice of $A_{0}$. We claim that $\varphi^{*} \in C_{\Sigma}\left(\operatorname{Hom}\left(\mathbf{A}_{1}, \underline{\mathbf{M}}\right), M\right)$. For suppose $r \in \Sigma$ is $n$-ary, $h_{1}, \ldots, h_{n} \in \operatorname{Hom}\left(\mathbf{A}_{1}, \underline{\mathbf{M}}\right)$, and $\left\langle\left(h_{1}(x), \ldots, h_{n}(x)\right\rangle \in r\right.$ for all $x \in A_{1}$. 
Let $g_{i}=\left.h_{i}\right|_{A_{0}}$ for $i=1, \ldots, n$; thus $(r, \bar{g}) \in U$, and there exist extensions of the $g_{i}$ to $\mathbf{A}_{(r, \bar{g})}$ (namely, $\left.h_{i}\right|_{\left.A_{(r, \bar{g}}\right)}$ ) which satisfy $r$ coordinatewise. Thus by the construction of $\mathbf{A}_{(r, \bar{g})}$ there exist $h_{1}^{\prime}, \ldots, h_{n}^{\prime} \in \operatorname{Hom}(\mathbf{A}, \underline{\mathbf{M}})$ extending $g_{1}, \ldots, g_{n}$ such that $\bar{h}^{\prime}(x) \in r$ for all $x \in A$. Then by definition of $\varphi^{*}$ we have $\left\langle\varphi^{*}\left(h_{1}\right), \ldots, \varphi^{*}\left(h_{n}\right)\right\rangle=\left\langle\varphi\left(h_{1}^{\prime}\right), \ldots, \varphi\left(h_{n}^{\prime}\right)\right\rangle \in r$ as $\varphi$ preserves $r$. This proves $\varphi^{*}$ preserves every $r \in \Sigma$, and $\varphi^{*}$ is automatically continuous as its domain is finite. So $\varphi^{*} \in C_{\Sigma}\left(\operatorname{Hom}\left(\mathbf{A}_{1}, \underline{\mathbf{M}}\right), M\right)$.

Since $\Sigma$ dualizes $\mathbf{A}_{1} \in \mathbb{I S P}_{\text {fin }}(\underline{\mathbf{M}})$, there exists $a \in A_{1}$ such that $\varphi^{*}(h)=h(a)$ for all $h \in \operatorname{Hom}\left(\mathbf{A}_{1}, \underline{\mathbf{M}}\right)$. It follows that $\varphi\left(h^{\prime}\right)=h^{\prime}(a)$ for all $h^{\prime} \in \operatorname{Hom}(\mathbf{A}, \underline{\mathbf{M}})$, i.e., $\varphi=e^{\mathbf{A}}(a)$. This proves that $e^{\mathbf{A}}$ is surjective and hence $\Sigma$ dualizes $\mathbb{I S P}(\underline{\mathbf{M}})$.

We now turn to full dualizability, first summarizing what has been known up until now. The condition in Definition 4.4(1) was isolated by Clark and Krauss in [5] (where it was called hull-kernel closure).

DEFINITION 4.4. Let $\underline{\mathbf{M}}$ be a finite algebra, $U$ a nonempty set, and $X \subseteq M^{U}$. Also let $\Sigma$ be a set of finitary operations and relations algebraic over $\underline{\mathbf{M}}$, and let $\mathbf{\sim}$ be the corresponding topological structure (see the introduction).

1. The term-closure of $X$, denoted by $[X]$, is the set of all $g \in M^{U}$ which satisfy the following property: for all $n<\omega, \bar{u} \in U^{n}$ and $n$-ary term operations $s(\bar{x}), t(\bar{x})$ of $\underline{\mathbf{M}}$, if $s\left(f\left(u_{1}\right), \ldots, f\left(u_{n}\right)\right)=t\left(f\left(u_{1}\right), \ldots, f\left(u_{n}\right)\right)$ for all $f \in X$, then $s\left(g\left(u_{1}\right), \ldots, g\left(u_{n}\right)\right)=t\left(g\left(u_{1}\right), \ldots, g\left(u_{n}\right)\right)$.

2. $X$ is term-closed if $X=[X]$.

3. TC $(\Sigma, \underline{\mathbf{M}})$ is the assertion that every topologically closed substructure of a power of $\underset{\sim}{\mathbf{M}}$ is term-closed. $\mathrm{TC}_{\text {fin }}(\Sigma, \underline{\mathbf{M}})$ is the restriction of this assertion to substructures of finite powers of $\mathbf{M}$.

4. $\Sigma$ strongly dualizes $\mathbb{I S P}(\underline{\mathbf{M}})$ if $\Sigma$ dualizes $\operatorname{ISP}(\underline{\mathbf{M}})$ and $\operatorname{TC}(\Sigma, \underline{\mathbf{M}})$ holds.

DEFINITION 4.5. Let $\underline{\mathbf{M}}$ be a finite algebra, and $\Sigma$ a set of finitary operations and relations algebraic over $\underline{\mathbf{M}}$.

1. By the partial clone generated by $\Sigma$ we mean the smallest set of finitary operations algebraic over $\underline{\mathbf{M}}$ which contains the projections and the operations in $\Sigma$, and is closed under all compositions for which the induced domain (by convention, the maximum possible) is nonempty.

2. GenClon $(\Sigma, \underline{\mathbf{M}})$ is the assertion that for every finitary operation $h$ algebraic over $\underline{\mathbf{M}}$ there exists an operation $h^{\prime}$ in the partial clone generated by $\Sigma$ having the same arity as $h$ and such that $\operatorname{dom}(h) \subseteq \operatorname{dom}\left(h^{\prime}\right)$ and $h=\left.h^{\prime}\right|_{\operatorname{dom}(h)}$.

3. $\operatorname{TC}(\underline{\mathbf{M}})$ is the assertion that $\operatorname{TC}(\nabla, \underline{\mathbf{M}})$ holds where $\nabla$ is the set of all finitary operations algebraic over $\underline{\mathbf{M}}$. 
LEMMA 4.6. Let $\underline{\mathbf{M}}$ be a finite algebra, and $\Sigma$ a set of finitary operations and relations algebraic over $\underline{\mathbf{M}}$.

1. If $\Sigma$ strongly dualizes $\mathbb{I S P}(\underline{\mathbf{M}})$, then $\Sigma$ fully dualizes $\mathbb{I S P}(\underline{\mathbf{M}})$.

2. $\mathrm{TC}(\Sigma, \underline{\mathbf{M}})$ is equivalent to the conjunction of $\mathrm{TC}(\underline{\mathbf{M}})$ and $\mathrm{TC}_{\mathrm{fin}}(\Sigma, \underline{\mathbf{M}})$.

3. $\operatorname{TC}_{\text {fin }}(\Sigma, \underline{\mathbf{M}})$ is equivalent to GenClon $(\Sigma, \underline{\mathbf{M}})$.

Proof. Clark and Krauss [5, page 21] proved item (1) (see also [2], Theorem 3.2.4). Item 2 is essentially Theorem 3.2.2 of [2]. Item 3 was proved by Davey, Haviar and Priestley as Theorem 5.3 in [7]; the result also appears as Lemma 9.4.1 of [2].

This completes our summary of the current understanding of full dualizability. Our aim now is to give a new sufficient condition for $\mathrm{TC}(\underline{\mathbf{M}})$ to hold. First we give another characterization of the condition $\operatorname{TC}(\Sigma, \underline{\mathbf{M}})$.

DEFINITION 4.7. Let $\underline{\mathbf{M}}$ be a finite algebra, $\Sigma$ a set of finitary operations and relations algebraic over $\underline{\mathbf{M}}$, and $\underset{\sim}{\mathbf{M}}$ the corresponding topological structure.

1. If $A \neq \varnothing$ and $X \subseteq M^{A}$, then we say $X$ separates the points of $A$ if for all $a, b \in A$ with $a \neq b$ there exists $f \in X$ with $f(a) \neq f(b)$.

2. SepGen $(\Sigma, \underline{\mathbf{M}})$ is the assertion that for every $\mathbf{A} \in \mathbb{I S P}(\underline{\mathbf{M}})$, if $X \subseteq \operatorname{Hom}(\mathbf{A}, \underline{\mathbf{M}})$ is a topologically closed substructure of ${\underset{\mathbf{M}}{\sim}}^{A}$ which separates the points of $A$, then $X=\operatorname{Hom}(\mathbf{A}, \underline{\mathbf{M}})$.

3. $\operatorname{ProjGen}(\Sigma, \underline{\mathbf{M}})$ is the assertion that for every $\mathbf{A} \leq \underline{\mathbf{M}}^{U}(U \neq \varnothing)$, if $X \subseteq \operatorname{Hom}(\mathbf{A}, \underline{\mathbf{M}})$ is a topologically closed subuniverse of ${\underset{\mathbf{M}}{\sim}}^{A}$ which contains all the projections $\pi_{u}$ $(u \in U)$, then $X=\operatorname{Hom}(\mathbf{A}, \underline{\mathbf{M}})$.

LEMMA 4.8. For any finite algebra $\underline{\mathbf{M}}$ and set $\Sigma$ of finitary operations and relations algebraic over $\mathbf{M}$, the following are equivalent:

1. $\operatorname{TC}(\Sigma, \underline{\mathbf{M}})$.

2. $\operatorname{SepGen}(\Sigma, \underline{\mathbf{M}})$.

3. $\operatorname{ProjGen}(\Sigma, \underline{\mathbf{M}})$.

Proof. (1) $\Rightarrow$ (2). Assume $\mathrm{TC}(\Sigma, \underline{\mathbf{M}})$. Let $\mathbf{A} \in \mathbb{I S P}(\underline{\mathbf{M}})$ and $X \subseteq \operatorname{Hom}(\mathbf{A}, \underline{\mathbf{M}})$ be such that $X$ is a closed subuniverse of ${\underset{\mathbf{M}}{ }}^{A}$ which separates the points of $A$, and assume there exists $g \in \operatorname{Hom}(\mathbf{A}, \underline{\mathbf{M}}) \backslash X$. By TC $(\Sigma, \underline{\mathbf{M}})$ we get that $X$ is term-closed, so there must exist $n<\omega,\left(a_{1}, \ldots, a_{n}\right) \in A^{n}$, and $n$-ary term operations $s$ and $t$ of $\underline{\mathbf{M}}$ witnessing the failure of $g$ to be in the term-closure of $X$.

Let $b=s\left(a_{1}, \ldots, a_{n}\right) \in A$ and $c=t\left(a_{1}, \ldots, a_{n}\right) \in A$. Then for all $f \in X$ we have $f(b)=f\left(s\left(a_{1}, \ldots, a_{n}\right)\right)=s\left(f\left(a_{1}\right), \ldots, f\left(a_{n}\right)\right)=t\left(f\left(a_{1}\right), \ldots, f\left(a_{n}\right)\right)=\cdots=f(c)$, 
while by the same argument $g(b) \neq g(c)$. These facts contradict the assumption that $X$ separates the points of $A$.

(3) is a special case of (2).

(3) $\Rightarrow(1)$. We begin with an alternate characterization of term-closure. Let $U$ be a nonempty set and $X \subseteq M^{U}$. For each $u \in U$ let $\pi_{u}: X \rightarrow M$ be the projection onto coordinate $u$, let $U^{*}=\left\{\pi_{u}: u \in U\right\} \subseteq M^{X}$, and let $\mathbf{A}_{X}$ be the subalgebra of $\underline{\mathbf{M}}^{X}$ generated by $U^{*}$. Define $v: \operatorname{Hom}\left(\mathbf{A}_{X}, \underline{\mathbf{M}}\right) \rightarrow M^{U}$ by $v(g)(u)=g\left(\pi_{u}\right)$. The following lemma, which we leave as an exercise, is a slight generalization of [2, Theorem 1.1.3].

LEMMA 4.9. With $\underline{\mathbf{M}}$ and $X$ as above, $v$ is a bijection from $\operatorname{Hom}\left(\mathbf{A}_{X}, \underline{\mathbf{M}}\right)$ to $[X]$.

Now assume $\operatorname{ProjGen}(\Sigma, \underline{\mathbf{M}})$. To prove $\operatorname{TC}(\Sigma, \underline{\mathbf{M}})$, let $U \neq \varnothing$ and let $X$ be a closed subuniverse of $\underset{\sim}{\mathbf{M}^{U}}$. Define $\mathbf{A}_{X}$ and $v$ as in the discussion preceding Lemma 4.9, and let $X^{*}=v^{-1}(X) \subseteq \operatorname{Hom}\left(\mathbf{A}_{X}, \underline{\mathbf{M}}\right)$. Note that if $\pi_{x}: \mathbf{A}_{X} \rightarrow \underline{\mathbf{M}}(x \in X)$ is a coordinate projection, then for every $u \in U, v\left(\pi_{x}\right)(u)=\pi_{x}\left(\pi_{u}\right)=\pi_{u}(x)=x(u)$, proving $v\left(\pi_{x}\right)=x$ and hence $\pi_{x} \in X^{*}$. It can be checked that $X^{*}$ is a closed subuniverse of ${\underset{\mathbf{M}}{ }}^{A_{X}}$. Thus $X^{*}=\operatorname{Hom}\left(\mathbf{A}_{X}, \underline{\mathbf{M}}\right)$ by $\operatorname{ProjGen}(\Sigma, \underline{\mathbf{M}})$, and so $X$ is equal to its term-closure by Lemma 4.9. This proves $\mathrm{TC}(\Sigma, \underline{\mathbf{M}})$.

We remark in passing that the above proof also gives the equivalence of $\operatorname{TC}_{\mathrm{fin}}(\Sigma, \underline{\mathbf{M}})$ with the restrictions of $\operatorname{ProjGen}(\Sigma, \underline{\mathbf{M}})$ and $\operatorname{SepGen}(\Sigma, \underline{\mathbf{M}})$ to finite subpowers $\mathbf{A}$ of $\underline{\mathbf{M}}$.

We now give our sufficient condition for $\mathrm{TC}(\underline{\mathbf{M}})$ to hold. We shall use this condition in Section 7. The condition has been generalized in [19] and [15].

DEFINITION 4.10. Suppose $\mathbf{A}_{0} \leq \mathbf{A}_{1} \leq \underline{\mathbf{M}}^{X}, h_{0} \in \operatorname{Hom}\left(\mathbf{A}_{0}, \underline{\mathbf{M}}\right)$, and $Y \subseteq X$.

1. $\left.\mathbf{A}_{1}\right|_{Y}$ denotes the image of $\mathbf{A}_{1}$ under the natural projection homomorphism $\pi_{Y}$ : $\mathbf{M}^{X} \rightarrow \underline{\mathbf{M}}^{Y}$.

2. We say that $h_{0}$ lifts to $\left.\mathbf{A}_{1}\right|_{Y}$ if there exists $h^{\prime} \in \operatorname{Hom}\left(\left.\mathbf{A}_{1}\right|_{Y}, \underline{\mathbf{M}}\right)$ such that $h_{0}(a)=$ $h^{\prime}\left(\left.a\right|_{Y}\right)$ for all $a \in A_{0}$.

THEOREM 4.11. Let $\underline{\mathbf{M}}$ be a finite algebra. Suppose there is a function $f: \omega \rightarrow \omega$ such that the following holds:

Whenever $\mathbf{A}_{0} \leq \mathbf{A}_{1} \leq \underline{\mathbf{M}}^{X}$ and $h_{0} \in \operatorname{Hom}\left(\mathbf{A}_{0}, \underline{\mathbf{M}}\right)$ where $X$ is finite and $h_{0}$ lifts to $\mathbf{A}_{1}$, then there exists $Y \subseteq X$ such that $|Y| \leq f\left(\left|A_{0}\right|\right)$ and $h_{0}$ lifts to $\left.\mathbf{A}_{1}\right|_{Y}$.

Then $\mathrm{TC}(\underline{\mathbf{M}})$ holds.

Proof. By Lemma 4.8, it suffices to prove $\operatorname{SepGen}(\nabla, \underline{\mathbf{M}})$ where $\nabla$ is the set of all finitary operations algebraic over $\underline{\mathbf{M}}$. Let $\underset{\sim}{\mathbf{M}}$ be the topological structure defined relative 
to the set $\nabla$. Let $f$ be the function given in the statement of the theorem. Note that the displayed condition remains true when $X$ is infinite, provided that $\mathbf{A}_{1}$ is finite.

Let $\mathbf{A} \in \mathbb{I S P}(\underline{\mathbf{M}})$ and suppose $X \subseteq \operatorname{Hom}(\mathbf{A}, \underline{\mathbf{M}})=: H$ where $X$ is a closed substructure of ${\underset{\mathbf{M}}{ }}^{A}$ which separates the points of $A$. It must be shown that $X=\operatorname{Hom}(\mathbf{A}, \underline{\mathbf{M}})$. Recall the natural embedding $e^{\mathbf{A}}: \mathbf{A} \hookrightarrow \underline{\mathbf{M}}^{H}$ defined prior to Lemma 3.2. Define $e^{*}: \mathbf{A} \hookrightarrow \underline{\mathbf{M}}^{X}$ by $e^{*}(a)=\left.e^{\mathbf{A}}(a)\right|_{X}$ and let $\mathbf{A}^{*}=\operatorname{ran}\left(e^{*}\right) \leq \underline{\mathbf{M}}^{X}$. If $\mathbf{B} \leq \mathbf{A}$ and $g \in \operatorname{Hom}(\mathbf{B}, \underline{\mathbf{M}})$ then we define $\mathbf{B}^{*}=e^{*}(\mathbf{B})$ and $g^{*}=g \circ\left(\left.e^{*}\right|_{B}\right)^{-1} \in \operatorname{Hom}\left(\mathbf{B}^{*}, \underline{\mathbf{M}}\right)$.

Fix $h \in \operatorname{Hom}(\mathbf{A}, \underline{\mathbf{M}})$. To show $h \in X$, it suffices (by the topological closedness of $X$ ) to show that $\left.\left.h\right|_{A_{0}} \in X\right|_{A_{0}}$ for every finite subalgebra $\mathbf{A}_{0}$ of $\mathbf{A}$. So let $\mathbf{A}_{0}$ be fixed and let $P$ be the poset of all finite subalgebras of $\mathbf{A}$ which contain $\mathbf{A}_{0}$, ordered by inclusion. Put $n=f\left(\left|A_{0}\right|\right)$ and $h_{0}=\left.h\right|_{A_{0}}$, and consider $h_{0}^{*} \in \operatorname{Hom}\left(\mathbf{A}_{0}^{*}, \underline{\mathbf{M}}\right)$. Suppose $\mathbf{A}_{1} \in P$ is given; $h_{0}$ clearly can be lifted to $\mathbf{A}_{1}$, hence $h_{0}^{*}$ can be lifted to $\mathbf{A}_{1}^{*}$, and as $\mathbf{A}_{0}^{*} \leq \mathbf{A}_{1}^{*} \leq \underline{\mathbf{M}}^{X}$ it follows from the hypothesis of the theorem that there exists $Y \subseteq X$ such that $|Y| \leq n$ and $h_{0}^{*}$ lifts to $\left.\mathbf{A}_{1}^{*}\right|_{Y}$. Enumerate $Y=\left\{y_{1}, \ldots, y_{s}\right\}$ with $s \leq n$. The latter condition $\left(h_{0}^{*}\right.$ lifting to $\left.\mathbf{A}_{1}^{*}\right|_{Y}$ ) can be restated as follows: there is an $s$-ary operation $g \in \nabla$ such that

1. $\left\langle y_{1}(a), \ldots, y_{s}(a)\right\rangle$ is in the domain of $g$ for every $a \in A_{1}$.

2. If $h^{\prime} \in \operatorname{Hom}\left(\mathbf{A}_{1}, \underline{\mathbf{M}}\right)$ is defined by $h^{\prime}(a)=g\left(y_{1}(a), \ldots, y_{s}(a)\right)$, then $\left.h^{\prime}\right|_{A_{0}}=h_{0}$.

For each $\mathbf{A}_{1} \in P$ let $F\left(\mathbf{A}_{1}\right)$ be the set of all triples $(s, \bar{y}, g)$ where $1 \leq s \leq n, \bar{y} \in\left(\left.X\right|_{A_{1}}\right)^{s}$, and $g \in \nabla$ is an $s$-ary operation satisfying items $(1,2)$ above with respect to $\bar{y} . F\left(\mathbf{A}_{1}\right)$ is finite and nonempty, by the above discussion. Moreover, if $\mathbf{A}_{1}, \mathbf{A}_{2} \in P$ with $\mathbf{A}_{1} \leq \mathbf{A}_{2}$, then $\left(s,\left.\bar{y}\right|_{A_{1}}, g\right) \in F\left(\mathbf{A}_{1}\right)$ whenever $(s, \bar{y}, g) \in F\left(\mathbf{A}_{2}\right)$. Thus by Lemma 4.1 there exist $s \leq n, \bar{y} \in\left(M^{A}\right)^{s}$, and an $s$-ary operation $g \in \nabla$ such that $\left(s,\left.\bar{y}\right|_{A_{1}}, g\right) \in F\left(\mathbf{A}_{1}\right)$ for all $\mathbf{A}_{1} \in P$. Define $h^{\prime} \in M^{A}$ by $h^{\prime}(a)=g\left(y_{1}(a), \ldots, y_{s}(a)\right)$. Since $X$ is topologically closed we get $y_{1}, \ldots, y_{s} \in X$. Then since $X$ is closed under $g$ we get $h^{\prime} \in X$. As $\left.h^{\prime}\right|_{A_{0}}=\left.h\right|_{A_{0}}$, this proves the theorem.

\section{Finite commutative local rings}

For the remainder of this paper all rings under consideration will have a named identity element. Saying that this element is named means for us that subrings are required to contain the identity element of the larger ring, and homomorphisms between rings are required to send the identity element of the one ring to the identity element of the other.

In this section we shall prove some facts about finite commutative rings with identity which are local, i.e., have a unique maximal ideal, and whose Jacobson radical is selfannihilating. If $R$ is such a ring then we denote the radical (= the unique maximal ideal) of $R$ by $J(R)$. For $n \in \mathbb{Z}$ we use $\mathbf{n}_{R}$ or just $\mathbf{n}$ for the corresponding element of $R$. The following facts are well-known and easily proved.

LEMMA 5.1. Let $R$ be a finite commutative local ring with identity such that $J(R)^{2}=\{0\}$. 
1. There exists a unique prime $p$ such that

(a) $\mathbf{p}_{R} \in J(R)$.

(b) $\operatorname{char}(R)=p$ or $p^{2}$ (depending on whether or not $\mathbf{p}_{R}=0$ ).

(c) $R / J(R)$ is a field of cardinality $p^{k}$ for some $k \geq 1$.

2. $\langle J(R),+\rangle$ is naturally a vector space over $R / J(R)$ via the scalar multiplication $(r+$ $J(R)) \cdot j=r j$.

3. The proper ideals of $R$ are precisely the vector subspaces of $J(R)$.

The unique prime $p$ in item 1 of the above lemma will sometimes be denoted by char* $(R)$. Suppose $R$ and $S$ are finite commutative local rings with identity and self-annihilating radicals such that $\operatorname{Hom}(S / J(S), R / J(R)) \neq \varnothing$; thus $\operatorname{char}^{*}(R)=\operatorname{char}^{*}(S)=p$ for some prime $p$. Let $K$ be the unique subfield of $R / J(R)$ of cardinality $|S / J(S)|$ and choose $\theta \in \operatorname{Hom}(S / J(S), R / J(R))$; thus $K$ is the image of $\theta$. Since $K$ is a subfield of $R / J(R)$, we can consider $J(R)$ as a vector space over $K$ in a natural way; by way of $\theta$, we can endow $J(R)$ with the structure of a vector space over $S / J(S)$. Let $J_{\theta}(R)$ denote this incarnation of $J(R)$, and let $\operatorname{Hom}_{\mathbf{p}}\left(J(S), J_{\theta}(R)\right)$ denote the set of all $S / J(S)$-linear transformations from $J(S)$ to $J_{\theta}(R)$ which send $\mathbf{p}_{S}$ to $\mathbf{p}_{R}$. Note that $\operatorname{Hom}_{\mathbf{p}}\left(J(S), J_{\theta}(R)\right) \neq \varnothing$ if and only if $\operatorname{char}(R) \mid \operatorname{char}(S)$.

LEMMA 5.2. Suppose $R$ and $S$ are finite commutative local rings with identity and self-annihilating radicals.

1. Every $h \in \operatorname{Hom}(S, R)$ induces an embedding $h^{*} \in \operatorname{Hom}(S / J(S), R / J(R))$ by the rule $h^{*}(s+J(S))=h(s)+J(R)$.

2. Suppose $\theta \in \operatorname{Hom}(S / J(S), R / J(R))$ and $h \in \operatorname{Hom}(S, R)$ such that $h^{*}=\theta$, and let $p=\operatorname{char}^{*}(R)=\operatorname{char}^{*}(S)$. Then $\left.h\right|_{J(S)} \in \operatorname{Hom}_{\mathbf{p}}\left(J(S), J_{\theta}(R)\right)$.

3. If $\operatorname{Hom}(S, R) \neq \varnothing$, then $\operatorname{Hom}(S / J(S), R / J(R)) \neq \varnothing$, $\operatorname{char}^{*}(S)=\operatorname{char}^{*}(R)$, and $\operatorname{char}(R) \mid \operatorname{char}(S)$.

If $R$ and $S$ are as in Lemma 5.2 and $\theta \in \operatorname{Hom}(S / J(S), R / J(R))$, then we define $\operatorname{Hom}_{\theta}(S, R)=\left\{h \in \operatorname{Hom}(S, R): h^{*}=\theta\right\}$. The previous lemma can be restated as follows:

1. $\operatorname{Hom}(S, R)=\bigcup\left\{\operatorname{Hom}_{\theta}(S, R): \theta \in \operatorname{Hom}(S / J(S), R / J(R))\right\}$.

2. For each $\theta \in \operatorname{Hom}(S / J(S), R / J(R))$, the map $v_{\theta}:\left.h \mapsto h\right|_{J(S)} \operatorname{defined~on} \operatorname{Hom}_{\theta}(S, R)$ sends $\operatorname{Hom}_{\theta}(S, R)$ into $\operatorname{Hom}_{\mathbf{p}}\left(J(S), J_{\theta}(R)\right)$.

Our next goal is to prove that the maps $v_{\theta}$ are bijections, and that the converse to Lemma 5.2(3) is true.

DEFINITION 5.3. Let $R$ be a finite commutative local ring with identity such that $J(R)^{2}=\{0\}$, and let $p=\operatorname{char}^{*}(R)$. Let $Q(R)$ denote the image in $R$ of the operation $t(x)=x^{p}$. 
CLAIM 5.4. Let $R$ and $p$ be as in the previous definition.

1. If $a, b \in R$, then $a \equiv b \bmod J(R)$ if and only if $a^{p}=b^{p}$. In particular, $a \in J(R)$ if and only if $a^{p}=0$.

2. $Q(R)$ intersects each coset of $J(R)$ at exactly one element.

3. $Q(R)$ is closed under multiplication; hence $\langle Q(R), \cdot\rangle$ "is" the multiplicative semigroup of the field $R / J(R)$.

LEMMA 5.5. Suppose $F(x) \in \mathbb{Z}_{p}[x]$ is monic and of degree $\ell$. Then there exists $F^{\star}(x) \in \mathbb{Z}[x]$, also monic and of degree $\ell$, such that

1. The image of $F^{\star}(x)$ under the canonical homomorphism $\mathbb{Z}[x] \rightarrow \mathbb{Z}_{p}[x]$ is $F(x)$.

2. In any commutative ring whose characteristic divides $p^{2}$, if an element a satisfies $a^{p^{\ell}}=a$ and $F^{\star}(a)^{p}=p F^{\star}(a)=0$, then $F^{\star}(a)=0$.

Proof. Begin by arbitrarily choosing $F_{1}(x) \in \mathbb{Z}[x]$ to be monic, of degree $\ell$, and satisfying item 1. Choose $G(x) \in \mathbb{Z}[x]$ so that $F_{1}\left(x^{p^{\ell}}\right)-F_{1}(x)^{p^{\ell}}=p G(x)$. As $F_{1}(x)$ is monic, we can write $G(x)=F_{1}(x) q(x)+r(x)$ where $q(x), r(x) \in \mathbb{Z}[x]$ and $\operatorname{deg}(r(x))<\ell$. Define $F^{\star}(x)=F_{1}(x)-\operatorname{pr}(x)$.

To prove this works, first note that $F^{\star}(x)$ is still monic, of degree $\ell$, and satisfies item 1 . Now suppose that $a$ is an element of a commutative ring whose characteristic divides $p^{2}$, and that $a^{p^{\ell}}=a$ and $F^{\star}(a)^{p}=p F^{\star}(a)=0$. Because of the assumption on the characteristic of the ring, we have $F_{1}(a)^{p}=p F_{1}(a)=0$. Then

$$
\begin{aligned}
F^{\star}(a) & =F_{1}(a)-p\left(G(a)-F_{1}(a) q(a)\right) \\
& =F_{1}(a)-\left(F_{1}\left(a^{p^{\ell}}\right)-F_{1}(a)^{p^{\ell}}\right)+p F_{1}(a) q(a) \\
& =0 \quad \text { as } a^{p^{\ell}}=a .
\end{aligned}
$$

THEOREM 5.6. Suppose $R$ and $S$ are finite commutative local rings with identity and self-annihilating radicals, that $\operatorname{char}^{*}(R)=\operatorname{char}^{*}(S)=p$, that $\operatorname{char}(R) \mid \operatorname{char}(S)$, and that $\operatorname{Hom}(S / J(S), R / J(R)) \neq \varnothing$. Then for each $\theta \in \operatorname{Hom}(S / J(S), R / J(R))$, the map $v_{\theta}$ : $\left.h \mapsto h\right|_{J(S)}$ is a bijection from $\operatorname{Hom}_{\theta}(S, R)$ to $\operatorname{Hom}_{\mathbf{p}}\left(J(S), J_{\theta}(R)\right)$.

Proof. Choose $k$, $\ell$ so that $R / J(R) \cong G F\left(p^{k}\right)$ and $S / J(S) \cong G F\left(p^{\ell}\right)$; thus $\ell \mid k$. Let $K$ be the unique subfield of $R / J(R)$ of cardinality $p^{\ell}$, let $\alpha$ be a generator of $K$, and let $\alpha_{1}, \ldots, \alpha_{\ell}$ be the conjugates of $\alpha$ (including $\alpha$ ) over $\mathbb{Z}_{p}$ in $K$. For each $i=1, \ldots, \ell$ let $a_{i}$ be the unique element of $Q(R) \cap \alpha_{i}$. Let $F(x) \in \mathbb{Z}_{p}[x]$ be the minimal polynomial of $\alpha$ over $\mathbb{Z}_{p}$, and let $F^{\star}(x) \in \mathbb{Z}[x]$ be the polynomial given by Lemma 5.5. As $F\left(\alpha_{i}\right)=0$ in $R / J(R)$ we have $F^{\star}\left(a_{i}\right) \in J(R)$ and hence $F^{\star}\left(a_{i}\right)^{p}=\mathbf{p} F^{\star}\left(a_{i}\right)=0$. Furthermore, $a_{i}^{p^{\ell}}=a_{i}$ by Claim 5.4(3); thus $F^{\star}\left(a_{i}\right)=0$ for $i=1, \ldots, \ell$ by Lemma 5.5. 
Similarly, $F(x)$ has a root $\gamma$ in $S / J(S)$. Let $c$ be the unique element of $\gamma \cap Q(S)$. By the same argument as before, we get $F^{\star}(c)=0$. For $i=1, \ldots, \ell$ let $\theta_{i}$ be the unique isomorphism from $S / J(S)$ to $K$ sending $\gamma$ to $\alpha_{i}$. Thus $\left\{\theta_{1}, \ldots, \theta_{\ell}\right\}=\operatorname{Hom}(S / J(S), R / J(R))$. Furthermore, for $h \in \operatorname{Hom}(S, R)$ and $i=1, \ldots, \ell$ the following are equivalent: (1) $h \in \operatorname{Hom}_{\theta_{i}}(S, R)$; (2) $h(c)=a_{i}$.

Fix $i=1, \ldots, \ell$ and put $\theta=\theta_{i}$. Since $S$ is generated by $J(S) \cup\{c\}$, the map $v_{\theta}$ : $\operatorname{Hom}_{\theta}(S, R) \rightarrow \operatorname{Hom}_{\mathbf{p}}\left(J(S), J_{\theta}(R)\right)$ is injective. It remains to prove that it is surjective. To do this, let $\lambda \in \operatorname{Hom}_{\mathbf{p}}\left(J(S), J_{\theta}(R)\right)$ be given. It can be easily shown that $\lambda(f(c) b)=$ $f\left(a_{i}\right) \lambda(b)$ for all $f(x) \in \mathbb{Z}[x]$ and $b \in J(S)$. Now "define" $h: S \rightarrow R$ by

$$
h(f(c)+b)=f\left(a_{i}\right)+\lambda(b),
$$

where $b$ ranges over $J(S)$ and $f(x)$ ranges over $\mathbb{Z}[x]$. Proving this is well-defined boils down to showing that if $f(x) \in \mathbb{Z}[x]$ is such that $f(c) \in J(S)$, then $\lambda(f(c))=f\left(a_{i}\right)$. Argue as follows: let $f^{o}(x)$ be the image of $f(x)$ in $\mathbb{Z}_{p}[x]$. As $f(c) \in J(S)$ we have $f^{o}(\gamma)=0$ and hence $F(x) \mid f^{o}(x)$ in $\mathbb{Z}_{p}[x]$. Thus there exist $g(x), h(x) \in \mathbb{Z}[x]$ such that $f(x)=F^{\star}(x) g(x)+p h(x)$. Then

$$
\begin{aligned}
f\left(a_{i}\right) & =F^{\star}\left(a_{i}\right) g\left(a_{i}\right)+h\left(a_{i}\right) \mathbf{p}_{R} \\
& =h\left(a_{i}\right) \mathbf{p}_{R}
\end{aligned}
$$

and

$$
\begin{aligned}
f(c) & =F^{\star}(c) g(c)+h(c) \mathbf{p}_{S} \\
& =h(c) \mathbf{p}_{S} .
\end{aligned}
$$

Thus $\lambda(f(c))=\lambda\left(h(c) \mathbf{p}_{S}\right)=h\left(a_{i}\right) \lambda\left(\mathbf{p}_{S}\right)=h\left(a_{i}\right) \mathbf{p}_{R}=f\left(a_{i}\right)$, as required. Clearly $\left.h\right|_{J(S)}=\lambda$; the proof that $h \in \operatorname{Hom}_{\theta}(S, R)$ is left as an exercise.

COROLLARY 5.7. Let $R$ and $S$ be as in Theorem 5.6. If $\theta \in \operatorname{Hom}(S / J(S), R / J(R))$ and $h_{1}, h_{2}, h_{3} \in \operatorname{Hom}_{\theta}(S, R)$, then $h_{1}-h_{2}+h_{3} \in \operatorname{Hom}_{\theta}(S, R)$.

Proof. Choose $a_{1}, \ldots, a_{\ell} \in Q(R)$ and $c \in Q(S)$ as in the proof of Theorem 5.6, and fix $i$ such that $\theta=\theta_{i}$. Let $\lambda_{j}=\left.h_{j}\right|_{J(S)} \in \operatorname{Hom}_{\mathbf{p}}\left(J(S), J_{\theta}(R)\right), j=1,2,3$. As $\lambda:=$ $\lambda_{1}-\lambda_{2}+\lambda_{3} \in \operatorname{Hom}_{\mathbf{p}}\left(J(S), J_{\theta}(R)\right)$ it follows that there exists $h \in \operatorname{Hom}_{\theta}(S, R)$ such that $\left.h\right|_{J(S)}=\lambda$. Moreover, by the proof of Theorem 5.6, for all $f(x) \in \mathbb{Z}[x]$ and $b \in J(S)$ we have

$$
\begin{aligned}
h(f(c)+b) & =f\left(a_{i}\right)+\lambda(b) \\
& =\left(f\left(a_{i}\right)-f\left(a_{i}\right)+f\left(a_{i}\right)\right)+\left(\lambda_{1}(b)-\lambda_{2}(b)+\lambda_{3}(b)\right) \\
& =h_{1}(f(c)+b)-h_{2}(f(c)+b)+h_{3}(f(c)+b) ;
\end{aligned}
$$

in other words, $h=h_{1}-h_{2}+h_{3}$. 
These are the facts we will need to prove that a finite commutative ring with identity and self-annihilating radical is 4-dualizable. (We will do that in the next section.) To prove full 4-dualizability, we will need one more fact.

LEMMA 5.8. Suppose $S$ is a finite commutative local ring with identity such that $J(S)^{2}=\{0\}$. Let $p=\operatorname{char}^{*}(S)$, and suppose $V, W$ are subspaces of $J(S)$ (i.e., proper ideals of $S)$ such that $V \oplus W=J(S)$ and $\operatorname{Span}\left(\mathbf{p}_{S}\right) \subseteq W$. Recall that $Q(S)$ denotes the image of $t(x)=x^{p}$ in $S$. Then there is an isomorphism $\varphi$ from $S / V$ to a subring

$T$ of $S$ such that $W \cup Q(S) \subseteq T$ and $S \stackrel{\text { nat }}{\rightarrow} S / V \stackrel{\varphi}{\cong} T$ is a retraction.

Proof. Choose $c$ (and $\ell, K, \alpha, F^{\star}$ etc.) as in the proof of Theorem 5.6, with $R=S$, but starting with the assumption that $\alpha$ is a primitive element of $K$ (equivalently, that $c$ is a generator of the cyclic group $\langle Q(S) \backslash\{0\}, \cdot\rangle)$. "Define" $\varphi: S / V \rightarrow S$ by

$$
\varphi((f(c)+w)+V)=f(c)+w
$$

where $w$ ranges over $W$ and $f(x)$ ranges over $\mathbb{Z}[x]$. Proving that $\varphi$ is well-defined boils down to showing that if $f(x) \in \mathbb{Z}[x]$ is such that $f(c) \in J(S)$, then $f(c) \in W$. In fact, if $f(c) \in J(S)$ then it was shown in the proof of Theorem 5.6 that $f(c) \in \operatorname{Span}\left(\mathbf{p}_{S}\right)$, which suffices. The proof that $\varphi$ is a ring homomorphism is left as an exercise. Since every element of $W \cup Q(S)$ can be represented as $0+w$ or $c^{n}+0$, we get $W \cup Q(S)$ included in the image of $\varphi$.

Suppose that $S_{0} \leq S \leq \prod_{x \in X} R_{x}$, that $h_{0} \in \operatorname{Hom}\left(S_{0}, R\right)$, and that $Y \subseteq X$. Let $\left.S\right|_{Y}$ denote the image of $S$ under the natural projection $\prod_{x \in X} R_{x} \rightarrow \prod_{x \in Y} R_{x}$. We say that $h_{0}$ lifts to $\left.S\right|_{Y}$ if there exists $h^{\prime} \in \operatorname{Hom}\left(\left.S\right|_{Y}, R\right)$ such that $h_{0}(a)=h^{\prime}\left(\left.a\right|_{Y}\right)$ for all $a \in S_{0}$.

COROLLARY 5.9. Suppose $S_{0} \leq S \leq \prod_{x \in X} R_{x}$ and $h_{0} \in \operatorname{Hom}\left(S_{0}, R\right)$, where $R$ and $S$ are finite commutative local rings with identity and self-annihilating radicals. If $h_{0}$ lifts to $S$, then there exists $Y \subseteq X$ with $|Y| \leq\left|S_{0}\right| \cdot|R / J(R)|$ such that $h_{0}$ lifts to $\left.S\right|_{Y}$.

Proof. Put $p=\operatorname{char}^{*}(S)$ and let $\operatorname{span}(\mathbf{p})$ be the span of $\mathbf{p}$ in $J(S)$ as a vector space over $S / J(S)$. Note that $|\operatorname{span}(\mathbf{p})| \leq|S / J(S)| \leq|R / J(R)|$, since $\operatorname{Hom}(S, R) \neq \varnothing$ (as $h_{0}$ lifts to $S)$. Now choose $Y \subseteq X$ large enough so that if $a \in\left(J\left(S_{0}\right)+\operatorname{span}(\mathbf{p})\right) \backslash\{0\}$ then $\left.a\right|_{Y} \neq 0$. Such $Y$ can be chosen with $|Y| \leq\left|J\left(S_{0}\right)+\operatorname{span}(\mathbf{p})\right| \leq\left|J\left(S_{0}\right)\right| \cdot|\operatorname{span}(\mathbf{p})| \leq\left|S_{0}\right| \cdot|R / J(R)|$. We claim that $h_{0}$ can be lifted to $\left.S\right|_{Y}$. To see this, first choose $h \in \operatorname{Hom}(S, R)$ such that $\left.h\right|_{S_{0}}=h_{0}$. Next, let $V$ be the kernel of $\left.\right|_{Y}:\left.S \rightarrow S\right|_{Y}$; thus $V$ is a subspace of $J(S)$ and $V \cap\left(J\left(S_{0}\right)+\operatorname{span}(\mathbf{p})\right)=\{0\}$. Choose a subspace $W$ of $J(S)$ such that $V \oplus W=J(S)$ and $J\left(S_{0}\right)+\operatorname{span}(\mathbf{p}) \subseteq W$.

By the previous lemma, there is an isomorphism $\varphi$ from $\left.S\right|_{Y}$ to a subring $T$ of $S$ such that $W \cup Q(S) \subseteq T$ and $\varphi\left(\left.a\right|_{Y}\right)=a$ for all $a \in T$. By Claim 5.4(2), since $S_{0}$ is itself local, 
every element of $S_{0}$ can be written in the form $z+b$ with $z \in Q\left(S_{0}\right)$ and $b \in J\left(S_{0}\right)$. Since $Q\left(S_{0}\right) \cup J\left(S_{0}\right) \subseteq Q(S) \cup W \subseteq T$ we have $S_{0} \leq T$. Thus if $h^{\prime}:\left.S\right|_{Y} \rightarrow R$ is defined by $h^{\prime}=h \circ \varphi$, then $h^{\prime}\left(\left.a\right|_{Y}\right)=h_{0}(a)$ for all $a \in S_{0}$ and therefore $h_{0}$ lifts to $\left.S\right|_{Y}$.

\section{Dualizing $\mathbb{I S P}(\underline{\mathbf{R}})$}

We revert to the notation appropriate for natural duality theory. Throughout this section, $\underline{\mathbf{R}}$ is a fixed finite commutative ring with identity whose radical is self-annihilating. Let $\bar{D}=\left\{(a, b, c) \in R^{3}: a \equiv b \equiv c(\bmod J(\underline{\mathbf{R}}))\right\}$ a subring of $\underline{\mathbf{R}}^{3}$. Note that the ternary partial operation $x-y+\left.z\right|_{D}$ is a homomorphism from $\mathbf{D}$ to $\underline{\mathbf{R}}$, because $J(\underline{\mathbf{R}})^{2}=\{0\}$, and hence is algebraic over $\underline{\mathbf{R}}$. Let $\mathcal{R}_{3}$ be the set of all ternary relations algebraic over $\underline{\mathbf{R}}$ and put $\Sigma_{0}=\mathcal{R}_{3} \cup\left\{x-y+\left.z\right|_{D}\right\}$.

\section{THEOREM 6.1. $\Sigma_{0}$ dualizes $\mathbb{I S P}(\underline{\mathbf{R}})$.}

Proof. Since $\Sigma_{0}$ is finite it suffices, by Theorem 4.3, to show the following: if $\mathbf{S} \in$ $\mathbb{I S P}_{\text {fin }}(\underline{\mathbf{R}})$ and $\varphi: \operatorname{Hom}(\mathbf{S}, \underline{\mathbf{R}}) \rightarrow R$ and $\varphi$ preserves each member of $\Sigma_{0}$, then there exists $b \in S$ such that $\varphi(h)=h(b)$ for all $h \in \operatorname{Hom}(\mathbf{S}, \underline{\mathbf{R}})$. If such $\mathbf{S}$ and $\varphi$ are given then, by Lemma 3.2(2), $\varphi$ preserves the relations in $\mathcal{R}_{3}$ if and only if

For all $h_{1}, h_{2}, h_{3} \in \operatorname{Hom}(\mathbf{S}, \underline{\mathbf{R}})$ there exists $b \in S$ such that $\varphi\left(h_{t}\right)=h_{t}(b)$ for $t=1,2,3$.

We shall refer to the displayed condition as 3-compatibility of $\varphi$.

Thus fix $\mathbf{S} \in \mathbb{I S P}_{\text {fin }}(\underline{\mathbf{R}})$ and $\varphi: \operatorname{Hom}(\mathbf{S}, \underline{\mathbf{R}}) \rightarrow R$ such that $\varphi$ is 3-compatible and preserves $x-y+\left.z\right|_{D}$. Decompose $\underline{\mathbf{R}}$ and $\mathbf{S}$ into directly indecomposable factors, say

$$
\begin{aligned}
\underline{\mathbf{R}} \cong \mathbf{R}_{1} \times \cdots \times \mathbf{R}_{k} \\
\mathbf{S} \cong \mathbf{S}_{1} \times \cdots \times \mathbf{S}_{\ell} .
\end{aligned}
$$

Also choose and fix appropriate projection homomorphisms $\pi_{i}^{R}: \underline{\mathbf{R}} \rightarrow \mathbf{R}_{i}(i=1, \ldots, k)$ and $\pi_{j}^{S}: \mathbf{S} \rightarrow \mathbf{S}_{j}(j=1, \ldots, \ell)$. Note that each factor $\mathbf{R}_{i}$ or $\mathbf{S}_{j}$ is local ([14], p. 76, Corollary 2) with self-annihilating radical, and that the radicals of $\underline{\mathbf{R}}$ and $\mathbf{S}$ are each the direct product of the radicals of their factors.

CLAIM 6.2. If $h, h^{\prime} \in \operatorname{Hom}(\mathbf{S}, \underline{\mathbf{R}})$ and $\pi_{i}^{R} h=\pi_{i}^{R} h^{\prime}$, then $\pi_{i}^{R}(\varphi(h))=\pi_{i}^{R}\left(\varphi\left(h^{\prime}\right)\right)$.

Proof. Using 3-compatibility of $\varphi$, choose $b \in S$ such that $\varphi(h)=h(b)$ and $\varphi\left(h^{\prime}\right)=$ $h^{\prime}(b)$. Then $\pi_{i}^{R}(\varphi(h))=\pi_{i}^{R} h(b)=\pi_{i}^{R} h^{\prime}(b)=\pi_{i}^{R}\left(\varphi\left(h^{\prime}\right)\right)$. 
Fix $i=1, \ldots, k$. As every $f \in \operatorname{Hom}\left(\mathbf{S}, \mathbf{R}_{i}\right)$ is of the form $f=\pi_{i}^{R} h$ for some $h \in \operatorname{Hom}(\mathbf{S}, \underline{\mathbf{R}})$, we can define $\varphi_{i}: \operatorname{Hom}\left(\mathbf{S}, \mathbf{R}_{i}\right) \rightarrow R_{i}$ by $\varphi_{i}\left(\pi_{i}^{R} h\right)=\pi_{i}^{R}(\varphi(h))$, where $h \in \operatorname{Hom}(\mathbf{S}, \underline{\mathbf{R}})$. Also define

$$
E_{i}=\left\{(a, b, c, d) \in\left(R_{i}\right)^{4}: a \equiv b \equiv c \equiv d \quad\left(\bmod J\left(\underline{\mathbf{R}}_{i}\right)\right) \text { and } a-b+c=d\right\} .
$$

CLAIM 6.3.

1. Each $\varphi_{i}$ preserves $E_{i}$.

2. $\varphi_{1}, \ldots, \varphi_{k}$ are mutually 3-compatible in the following sense: if $h_{t} \in \operatorname{Hom}\left(\mathbf{S}, \mathbf{R}_{i_{t}}\right)$, $t=1,2,3$, then there exists $b \in S$ such that $\varphi_{i_{t}}\left(h_{t}\right)=h_{t}(b)$ for $t=1,2,3$.

Proof. To prove that $\varphi_{i}$ preserves $E_{i}$, we must show that if $h_{1}, \ldots, h_{4} \in \operatorname{Hom}\left(\mathbf{S}, \mathbf{R}_{i}\right)$ are such that $\left(h_{1}(b), \ldots, h_{4}(b)\right) \in E_{i}$ for all $b \in S$, then $\left(\varphi_{i}\left(h_{1}\right), \ldots, \varphi_{i}\left(h_{4}\right)\right) \in E_{i}$. Given such $h_{1}, \ldots, h_{4}$, choose $h_{1}^{*}, \ldots, h_{4}^{*} \in \operatorname{Hom}(\mathbf{S}, \underline{\mathbf{R}})$ such that $\pi_{i}^{R} h_{t}^{*}=h_{t}$ for $t=1, \ldots, 4$ and $\pi_{j}^{R} h_{1}^{*}=\cdots=\pi_{j}^{R} h_{4}^{*}$ for all $j \neq i$. Since $(c, c, c, c) \in E_{j}$ for all $j$ and all $c \in R_{j}$, we have $\left(h_{1}^{*}(b), h_{2}^{*}(b), h_{3}^{*}(b)\right) \in D$ for all $b \in S$ and $h_{1}^{*}-h_{2}^{*}+h_{3}^{*}=h_{4}^{*}$. As $\varphi$ preserves $x-y+\left.z\right|_{D}$ we get $\left(\varphi\left(h_{1}^{*}\right), \varphi\left(h_{2}^{*}\right), \varphi\left(h_{3}^{*}\right)\right) \in D$ and $\varphi\left(h_{1}^{*}\right)-\varphi\left(h_{2}^{*}\right)+\varphi\left(h_{3}^{*}\right)=\varphi\left(h_{4}^{*}\right)$. Therefore $\left(\pi_{i}^{R} \varphi\left(h_{1}^{*}\right), \ldots, \pi_{i}^{R} \varphi\left(h_{4}^{*}\right)\right) \in E_{i}$. As $\pi_{i}^{R} \varphi\left(h_{t}^{*}\right)=\varphi_{i}\left(\pi_{i}^{R} h_{t}^{*}\right)=\varphi_{i}\left(h_{t}\right)$, we get $\left(\varphi_{i}\left(h_{1}\right), \ldots, \varphi_{i}\left(h_{4}\right)\right) \in E_{i}$ as desired.

Next suppose $h_{t} \in \operatorname{Hom}\left(\mathbf{S}, \mathbf{R}_{i_{t}}\right)$ for $t=1,2,3$. Choose $h_{t}^{*} \in \operatorname{Hom}(\mathbf{S}, \underline{\mathbf{R}})$ such that $\pi_{i_{t}}^{R} h_{t}^{*}=h_{t}$ for $t=1,2,3$. By 3-compatibility of $\varphi$ there exists $b \in S$ such that $\varphi\left(h_{t}^{*}\right)=$ $h_{t}^{*}(b)$ for $t=1,2,3$. Then $\varphi_{i_{t}}\left(h_{t}\right)=\varphi_{i_{t}}\left(\pi_{i_{t}}^{R} h_{t}^{*}\right)=\pi_{i_{t}}^{R}\left(\varphi\left(h_{t}^{*}\right)\right)=\pi_{i_{t}}^{R} h_{t}^{*}(b)=h_{t}(b)$ for each $t$.

Define the relation $P \subseteq\{1, \ldots, k\} \times\{1, \ldots, \ell\}$ by $i P j$ iff $\operatorname{Hom}\left(\mathbf{S}_{j}, \mathbf{R}_{i}\right) \neq \varnothing$. If $i P j$ define $\varphi_{i j}: \operatorname{Hom}\left(\mathbf{S}_{j}, \mathbf{R}_{i}\right) \rightarrow R_{i}$ by $\varphi_{i j}(h)=\varphi_{i}\left(h \pi_{j}^{S}\right)$.

\section{CLAIM 6.4.}

1. Each $\varphi_{i j}$ preserves $E_{i}$.

2. For each $j$, the family $\left\{\varphi_{i j}: i P j\right\}$ is mutually 3-compatible.

Proof. Exercise.

Now fix $j$ and let $\mathbf{S}^{*}=\mathbf{S}_{j}$ and $I=\{i: i P j\}$. Our next goal is to prove that if $\left\{\varphi_{i}^{*}: i \in I\right\}$ is any family of maps $\varphi_{i}^{*}: \operatorname{Hom}\left(\mathbf{S}^{*}, \mathbf{R}_{i}\right) \rightarrow R_{i}$ such that (i) each $\varphi_{i}^{*}$ preserves $E_{i}$, and (ii) $\left\{\varphi_{i}^{*}: i \in I\right\}$ is mutually 3-compatible, then there exists $b \in S^{*}$ such that for all $i \in I$ and all $h \in \operatorname{Hom}\left(\mathbf{S}^{*}, \mathbf{R}_{i}\right)$ we have $\varphi_{i}^{*}(h)=h(b)$. (In particular, this will be true of the family $\left\{\varphi_{i j}: i \in I\right\}$.) Suppose $\left\{\varphi_{i}^{*}: i \in I\right\}$ is such a family.

CLAIM 6.5. We may assume with no loss of generality that $\operatorname{ran}\left(\varphi_{i}^{*}\right) \subseteq J\left(\mathbf{R}_{i}\right)$ for each $i \in I$. 
Proof. Fix $i_{0} \in I$ and $f_{0} \in \operatorname{Hom}\left(\mathbf{S}^{*}, \mathbf{R}_{i_{0}}\right)$. By 3-compatibility choose $c \in S^{*}$ such that $\varphi_{i}^{*}\left(f_{0}\right)=f_{0}(c)$. For each $i \in I$ define $\psi_{i}: \operatorname{Hom}\left(\mathbf{S}^{*}, \mathbf{R}_{i}\right) \rightarrow R_{i}$ by $\psi_{i}(h)=\varphi_{i}^{*}(h)-h(c)$. Note in particular that $\psi_{i_{0}}\left(f_{0}\right)=0$.

It is easy to show that each $\psi_{i}$ preserves $E_{i}$. To show that $\left\{\psi_{i}: i \in I\right\}$ is mutually 3-compatible, simply note that if $i_{1}, i_{2}, i_{3} \in I$ and $h_{t} \in \operatorname{Hom}\left(\mathbf{S}^{*}, \mathbf{R}_{i_{t}}\right)$, for $t=1,2,3$, and $b \in S^{*}$ witnesses 3-compatibility for the $\varphi_{i}^{*}$ 's at $h_{1}, h_{2}, h_{3}$, then $b-c$ witnesses 3-compatibility for the $\psi_{i}$ 's at $h_{1}, h_{2}, h_{3}$.

Thus $\left\{\psi_{i}: i \in I\right\}$ is one of the families which we need to consider. Moreover, suppose $i \in I$ and $h \in \operatorname{Hom}\left(\mathbf{S}^{*}, \mathbf{R}_{i}\right)$. Using mutual 3-compatibility of the $\psi_{i}$ 's, choose $b \in S^{*}$ such that $\psi_{i}(h)=h(b)$ and $\psi_{i_{0}}\left(f_{0}\right)=f_{0}(b)$. As $\psi_{i_{0}}\left(f_{0}\right)=0$ we get $b \in \operatorname{ker}\left(f_{0}\right)$. Since $\operatorname{ker}\left(f_{0}\right) \subseteq J\left(\mathbf{S}^{*}\right)$ and $h\left(J\left(\mathbf{S}^{*}\right)\right) \subseteq J\left(\mathbf{R}_{i}\right)$ we get $\psi_{i}(h)=h(b) \in J\left(\mathbf{R}_{i}\right)$. Thus $\operatorname{ran}\left(\psi_{i}\right) \subseteq J\left(\mathbf{R}_{i}\right)$ for each $i \in I$.

Finally, $d \in S^{*}$ satisfies $\psi(h)=h(d)$ for all $i \in I$ and $h \in \operatorname{Hom}\left(\mathbf{S}^{*}, \mathbf{R}_{i}\right)$ if and only if $d+c$ satisfies $\varphi_{i}^{*}(h)=h(d+c)$ for all $i \in I$ and $h \in \operatorname{Hom}\left(\mathbf{S}^{*}, \mathbf{R}_{i}\right)$.

Thus we shall assume that the family $\left\{\varphi_{i}^{*}: i \in I\right\}$ has the additional property that $\operatorname{ran}\left(\varphi_{i}^{*}\right) \subseteq J\left(\mathbf{R}_{i}\right)$ for $i \in I$. For each $i \in I$ and $h \in \operatorname{Hom}\left(\mathbf{S}^{*}, \mathbf{R}_{i}\right)$ let $C_{h}=\left\{b \in S^{*}\right.$ : $\left.h(b)=\varphi_{i}^{*}(h)\right\} . C_{h}$ is nonempty by 3 -compatibility and hence is a coset of $\operatorname{ker}(h)$. Moreover, $\varphi_{i}^{*}(h) \in J\left(\mathbf{R}_{i}\right)$ implies $C_{h} \subseteq J\left(\mathbf{S}^{*}\right)$; that is, $C_{h}$ is a subspace of the vector space $J\left(\mathbf{S}^{*}\right)$ (over $\mathbf{S}^{*} / J\left(\mathbf{S}^{*}\right)$ ). Our goal, restated, is to prove that $\bigcap\left\{C_{h}: h \in \operatorname{Hom}\left(\mathbf{S}^{*}, \mathbf{R}_{i}\right), i \in I\right\}$ is nonempty.

CLAIM 6.6. If $h_{t} \in \operatorname{Hom}\left(\mathbf{S}^{*}, \mathbf{R}_{i_{t}}\right)$, for $t=1,2,3$, and $\operatorname{ker}\left(h_{1}\right) \cap \operatorname{ker}\left(h_{2}\right) \subseteq \operatorname{ker}\left(h_{3}\right)$, then $C_{h_{1}} \cap C_{h_{2}} \subseteq C_{h_{3}}$.

Proof. $C_{h_{1}} \cap C_{h_{2}} \cap C_{h_{3}} \neq \varnothing$ by mutual 3-compatibility, and $C_{h_{1}} \cap C_{h_{2}}$ is a coset of $\operatorname{ker}\left(h_{1}\right) \cap \operatorname{ker}\left(h_{2}\right)$; hence $C_{h_{1}} \cap C_{h_{2}} \subseteq C_{h_{3}}$.

Now put $p=\operatorname{char}^{*}\left(\mathbf{S}^{*}\right)$ and consider cases.

CASE 1. $\mathbf{p}_{S^{*}}=0$.

If $J\left(\mathbf{S}^{*}\right)=\{0\}$ then $C_{h}=\{0\}$ for all $i \in I$ and $h \in \operatorname{Hom}\left(\mathbf{S}^{*}, \mathbf{R}_{i}\right)$ and hence $0 \in \bigcap\left\{C_{h}\right.$ : $\left.h \in \operatorname{Hom}\left(\mathbf{S}^{*}, \mathbf{R}_{i}\right), i \in I\right\}$. So assume $J\left(\mathbf{S}^{*}\right) \neq \varnothing$. Let $\left\{e_{1}, \ldots, e_{m}\right\}$ be a basis for $J\left(\mathbf{S}^{*}\right)$ (as a vector space over $\mathbf{S}^{*} / J\left(\mathbf{S}^{*}\right)$ ), and for each $t=1, \ldots, m$ let $W_{t}=\operatorname{span}\left(\left\{e_{u}: u \neq t\right\}\right.$ ).

Recall that $\mathbf{S}$ is residually in $\left\{\mathbf{R}_{1}, \ldots, \mathbf{R}_{k}\right\}$, that $\mathbf{S}^{*}$ is a direct factor of $\mathbf{S}$, and that $\mathbf{R}_{1}, \ldots, \mathbf{R}_{k}$ are directly indecomposable. It is a property of rings with identity that this information implies $\mathbf{S}^{*}$ is residually in $\left\{\mathbf{R}_{1}, \ldots, \mathbf{R}_{k}\right\}$ and hence $\mathbf{S}^{*}$ is residually in $\left\{\mathbf{R}_{i}\right.$ : $i \in I\}$. It follows that there exists $i_{0} \in I$ such that $J\left(\mathbf{R}_{i_{0}}\right) \neq\{0\}$. By Theorem 5.6 there exist $f_{1}, \ldots, f_{m} \in \operatorname{Hom}\left(\mathbf{S}^{*}, \mathbf{R}_{i_{0}}\right)$ such that $\operatorname{ker}\left(f_{t}\right)=W_{t}$ for $t=1, \ldots, m$. Thus $C_{f_{1}} \cap \cdots \cap C_{f_{m}}$ is automatically nonempty; let $b$ be the unique element in the intersection. 
We claim that $b \in C_{h}$ for all $i \in I$ and $h \in \operatorname{Hom}\left(\mathbf{S}^{*}, \mathbf{R}_{i}\right)$. To prove this, let $i$ and $h$ be given, and choose $\theta \in \operatorname{Hom}\left(\mathbf{S}^{*} / J\left(\mathbf{S}^{*}\right), \mathbf{R}_{i} / J\left(\mathbf{R}_{i}\right)\right)$ such that $h \in \operatorname{Hom}_{\theta}\left(\mathbf{S}^{*}, \mathbf{R}_{i}\right)$. For each $t=1, \ldots, m$ let $\lambda_{t}$ be the unique member of $\operatorname{Hom}_{\mathbf{p}}\left(J\left(\mathbf{S}^{*}\right), J_{\theta}\left(\mathbf{R}_{i}\right)\right)$ satisfying

$$
\lambda_{t}\left(e_{u}\right)= \begin{cases}h\left(e_{t}\right) & \text { if } u=t \\ 0 & \text { otherwise. }\end{cases}
$$

Also let $\lambda_{0} \in \operatorname{Hom}_{\mathbf{p}}\left(J\left(\mathbf{S}^{*}\right), J_{\theta}\left(\mathbf{R}_{i}\right)\right)$ be the constant 0 map. For each $t=0, \ldots, m$ let $g_{t}$ be the unique member of $\operatorname{Hom}_{\theta}\left(\mathbf{S}^{*}, \mathbf{R}_{i}\right)$ such that $\left.g_{t}\right|_{J\left(\mathbf{S}^{*}\right)}=\lambda_{t}$, and define $h_{0}=g_{0}$ and $h_{t}=h_{t-1}-g_{0}+g_{t}$ for $1 \leq t \leq m$. Note that $h_{t} \in \operatorname{Hom}_{\theta}\left(\mathbf{S}^{*}, \mathbf{R}_{i}\right)$ for all $t$, by Corollary 5.7. Furthermore, $\left.h_{m}\right|_{J\left(\mathbf{S}^{*}\right)}=\left.h\right|_{J\left(\mathbf{S}^{*}\right)}$ and therefore $h_{m}=h$ by Theorem 5.6. We will show $b \in C_{h_{t}}$ for each $t$. Obviously $C_{h_{0}}=C_{g_{0}}=J\left(\mathbf{S}^{*}\right)$, so $b \in C_{h_{0}}=C_{g_{0}}$. Assume that $t>0$ and $b \in C_{h_{t-1}}$. Since $\operatorname{ker}\left(f_{t}\right)=W_{t} \subseteq \operatorname{ker}\left(g_{t}\right)$ we get $C_{f_{t}} \subseteq C_{g_{t}}$ by Claim 6.6 and hence $b \in C_{g_{t}}$. Thus

$$
\begin{aligned}
h_{t-1}(b) & =\varphi_{i}^{*}\left(h_{t-1}\right) \\
g_{0}(b) & =\varphi_{i}^{*}\left(g_{0}\right) \quad(=0) \\
g_{t}(b) & =\varphi_{i}^{*}\left(g_{t}\right) .
\end{aligned}
$$

Finally, note that $\left(h_{t-1}(x), g_{0}(x), g_{t}(x), h_{t}(x)\right) \in E_{i}$ for all $x \in \mathbf{S}^{*}$. As $\varphi_{i}^{*}$ preserves $E_{i}$ we have

$$
\begin{aligned}
\varphi_{i}^{*}\left(h_{t}\right) & =\varphi_{i}^{*}\left(h_{t-1}\right)-\varphi_{i}^{*}\left(g_{0}\right)+\varphi_{i}^{*}\left(g_{t}\right) \\
& =h_{t-1}(b)-g_{0}(b)+g_{t}(b) \\
& =h_{t}(b),
\end{aligned}
$$

proving $b \in C_{h_{t}}$. When $t=m$ this yields $b \in C_{h}$.

CASE 2. $\mathbf{p}_{S^{*}} \neq 0$.

Again, $\mathbf{S}^{*}$ is residually in $\left\{\mathbf{R}_{i}: i \in I\right\}$ and therefore there exists $i_{0} \in I$ such that $\mathbf{p}_{R_{i_{0}}} \neq 0$. Choose any $h_{0} \in \operatorname{Hom}\left(\mathbf{S}^{*}, \mathbf{R}_{i_{0}}\right)$. If $J\left(\mathbf{S}^{*}\right)=\operatorname{span}\left(\mathbf{p}_{S^{*}}\right)$ then $\operatorname{ker}\left(h_{0}\right)=\{0\}$ and therefore $C_{h_{0}}=\{b\}$ for some $b \in J\left(\mathbf{S}^{*}\right)$. In this case, if $i \in I$ and $h \in \operatorname{Hom}\left(\mathbf{S}^{*}, \mathbf{R}_{i}\right)$ are arbitrary, then $C_{h} \cap C_{h_{0}} \neq \varnothing$ by mutual 3-compatibility and thus we have $b \in C_{h}$ as desired.

So assume $J\left(\mathbf{S}^{*}\right) \neq \operatorname{span}\left(\mathbf{p}_{S^{*}}\right)$. Let $\left\{e_{0}, e_{1}, \ldots, e_{m}\right\}$ be a basis for $J\left(\mathbf{S}^{*}\right)$ such that $\sum_{i} e_{i}=\mathbf{p}_{S^{*}}$. For $t=0, \ldots, m$ let $W_{t}=\operatorname{span}\left(\left\{e_{u}: u \neq t\right\}\right)$. By Theorem 5.6, there exist $f_{0}, \ldots, f_{m} \in \operatorname{Hom}\left(\mathbf{S}^{*}, \mathbf{R}_{i_{0}}\right)$ such that $\operatorname{ker}\left(f_{t}\right)=W_{t}$ for $t=0, \ldots, m$. Thus $C_{f_{0}} \cap \cdots \cap$ $C_{f_{m}} \neq \varnothing$ automatically; let $b$ be the unique element in the intersection. We will show $b \in C_{h}$ for all $h \in \operatorname{Hom}\left(\mathbf{S}^{*}, \mathbf{R}_{i}\right), i \in I$. 
Let $i$ and $h$ be given. Choose $\theta$ so that $h \in \operatorname{Hom}_{\theta}\left(\mathbf{S}^{*}, \mathbf{R}_{i}\right)$. For $t=1, \ldots, m$ let $\lambda_{t}$ be the unique member of $\operatorname{Hom}_{\mathbf{p}}\left(J\left(\mathbf{S}^{*}\right), J_{\theta}\left(\mathbf{R}_{i}\right)\right)$ satisfying

$$
\lambda_{t}\left(e_{u}\right)= \begin{cases}h\left(e_{t}\right) & \text { if } u=t \\ \mathbf{p}_{R_{i}}-h\left(e_{t}\right) & \text { if } u=0 \\ 0 & \text { otherwise. }\end{cases}
$$

Also let $\lambda_{0}$ be the unique member of $\operatorname{Hom}_{\mathbf{p}}\left(J\left(\mathbf{S}^{*}\right), J_{\theta}\left(\mathbf{R}_{i}\right)\right)$ satisfying $\lambda_{0}\left(e_{0}\right)=\mathbf{p}_{R_{i}}$ while $\lambda_{0}\left(e_{u}\right)=0$ for $1 \leq u \leq m$. For each $t=0, \ldots, m$ let $g_{t}$ be the unique member of $\operatorname{Hom}_{\theta}\left(\mathbf{S}^{*}, \mathbf{R}_{i}\right)$ such that $\left.g_{t}\right|_{J\left(\mathbf{S}^{*}\right)}=\lambda_{t}$, and define $h_{0}=g_{0}$ and $h_{t}=h_{t-1}-g_{0}+g_{t}$ for $1 \leq t \leq m$. Note that $h_{t} \in \operatorname{Hom}_{\theta}\left(\mathbf{S}^{*}, \mathbf{R}_{i}\right)$ for all $t$, by Corollary 5.7. Furthermore, $\left.h_{m}\right|_{J\left(\mathbf{S}^{*}\right)}=\left.h\right|_{J\left(\mathbf{S}^{*}\right)}$ and therefore $h_{m}=h$ by Theorem 5.6. We will show $b \in C_{h_{t}}$ for each $t$. Note that $\operatorname{ker}\left(g_{0}\right)=W_{0}=\operatorname{ker}\left(f_{0}\right)$, so $C_{g_{0}}=C_{f_{0}}$ by Corollary 6.6. For $t>0$ we have $\operatorname{ker}\left(g_{t}\right) \supseteq W_{0} \cap W_{t}=\operatorname{ker}\left(f_{0}\right) \cap \operatorname{ker}\left(f_{t}\right)$ and therefore $C_{g_{t}} \supseteq C_{f_{0}} \cap C_{f_{t}}$ by Corollary 6.6. Thus $b \in C_{g_{t}}$ for all $t$. Hence the same argument given in Case 1 yields $b \in C_{h}$.

This completes the goal set out before Claim 6.5. As a consequence, we have proved the following: for each $j=1, \ldots, \ell$ there exists $b_{j} \in S_{j}$ such that for all $i=1, \ldots, k$, if $i P j$ (i.e., $\left.\operatorname{Hom}\left(\mathbf{S}_{j}, \mathbf{R}_{i}\right) \neq \varnothing\right)$ then $\varphi_{i j}(h)=h\left(b_{j}\right)$ for all $h \in \operatorname{Hom}\left(\mathbf{S}_{j}, \mathbf{R}_{i}\right)$. Let $b$ be the unique element of $\mathbf{S}$ such that $\pi_{j}^{S}(b)=b_{j}$ for $j=1, \ldots, \ell$. We will complete the proof of the theorem by showing

CLAIM 6.7. $\varphi(h)=h(b)$ for all $h \in \operatorname{Hom}(\mathbf{S}, \underline{\mathbf{R}})$.

It suffices to show $\varphi_{i}(h)=h(b)$ for all $i=1, \ldots, k$ and all $h \in \operatorname{Hom}\left(\mathbf{S}, \mathbf{R}_{i}\right)$. Fix $i$ and $h \in \operatorname{Hom}\left(\mathbf{S}, \mathbf{R}_{i}\right)$. As $\mathbf{R}_{i}$ is local, there must exist $j$ such that $\operatorname{ker}\left(\pi_{j}^{S}\right) \subseteq \operatorname{ker}(h)$. Let $h^{*} \in \operatorname{Hom}\left(\mathbf{S}_{j}, \mathbf{R}_{i}\right)$ be such that $h=h^{*} \pi_{j}^{S}$. Then $\varphi_{i}(h)=\varphi_{i}\left(h^{*} \pi_{j}^{S}\right)=\varphi_{i j}\left(h^{*}\right)=$ $h^{*}\left(b_{j}\right)=h^{*} \pi_{j}^{S}(b)=h(b)$, as required.

\section{Strongly dualizing $\mathbb{I S P}(\underline{\mathbf{R}})$}

We continue to assume that $\mathbf{R}$ is a finite commutative ring with identity whose radical is self-annihilating. Let $\mathbf{R}_{1}, \ldots, \mathbf{R}_{k}$ be the directly indecomposable factors of $\underline{\mathbf{R}}$ and let $\pi_{1}^{R}, \ldots, \pi_{k}^{R}$ be the corresponding projection homomorphisms from $\underline{\mathbf{R}}$ as in the previous section. Let $d: \underline{\mathbf{R}}^{k} \rightarrow \underline{\mathbf{R}}$ be the $k$-ary "decomposition homomorphism" defined so that $\pi_{i}^{R}(d(a))=\pi_{i}^{R}\left(a_{i}\right)$ for all $a \in \underline{\mathbf{R}}^{k}$ and all $i=1, \ldots, k$, that is,

$d\left(\left[a_{11}, \ldots, a_{1 k}\right],\left[a_{21}, \ldots, a_{2 k}\right], \ldots,\left[a_{k 1}, \ldots, a_{k k}\right]\right)=\left[a_{11}, a_{22}, \ldots, a_{k k}\right]$.

THEOREM 7.1. Let $\underline{\mathbf{R}}$ be a finite commutative ring with identity whose radical $J(\underline{\mathbf{R}})$ is self annihilating. Then $\mathbb{I S P}(\underline{\mathbf{R}})$ is strongly dualized by the set $\Sigma$ consisting of

1. the (partial) operation $x-y+\left.z\right|_{D}$, where $D=\left\{(x, y, z) \in R^{3}: x \equiv_{J} y \equiv_{J} z\right\}$,

2. the set $\mathcal{F}_{2}$ of all binary (partial) operations algebraic over $\underline{\mathbf{R}}$, 
3. the set $\mathcal{R}_{3}$ of all ternary relations algebraic over $\underline{\mathbf{R}}$, and

4. the $k$-ary decomposition operation $d$ defined above.

Proof. $\underline{\mathbf{R}}$. By Lemma 4.6 it will suffice to prove $\mathrm{TC}(\underline{\mathbf{R}})$ and $\operatorname{GenClon}(\Sigma, \underline{\mathbf{R}})$.

CLAIM 7.2. Suppose $\mathbf{S}_{0} \leq \mathbf{S} \leq \underline{\mathbf{R}}^{U}$ and $h_{0} \in \operatorname{Hom}\left(\mathbf{S}_{0}, \underline{\mathbf{R}}\right)$ where $U$ is finite and $h_{0}$ lifts to $\mathbf{S}$. There exists $Y \subseteq U$ with $|Y| \leq\left|S_{0}\right| \cdot|R / J(\underline{\mathbf{R}})|$ such that $h_{0}$ lifts to $\left.\mathbf{S}\right|_{Y}$.

Proof. Let $\mathbf{S}_{1}, \ldots, \mathbf{S}_{\ell}$ and $\pi_{1}^{S}, \ldots, \pi_{\ell}^{S}$ be chosen for $\mathbf{S}$ as in the proof of Theorem 6.1. For each $u \in U$ let $\rho_{u} \in \operatorname{Hom}(\mathbf{S}, \underline{\mathbf{R}})$ be the projection onto coordinate $u$. For each $j=1, \ldots, \ell$ let

$$
X_{j}=\left\{(u, s) \in U \times\{1, \ldots, k\}: \operatorname{ker}\left(\pi_{s}^{R} \rho_{u}\right) \subseteq \operatorname{ker}\left(\pi_{j}^{S}\right)\right\}
$$

Thus $X_{1}, \ldots, X_{\ell}$ partition $U \times\{1, \ldots, k\}$. For $(u, s) \in X_{j}$ define $\bar{\rho}_{u, s} \in \operatorname{Hom}\left(\mathbf{S}_{j}, \underline{\mathbf{R}}_{s}\right)$ so that $\bar{\rho}_{u, s} \pi_{j}^{S}=\pi_{s}^{R} \rho_{u}$. For $j=1, \ldots, \ell$ define $\bar{\rho}_{j}: \mathbf{S}_{j} \hookrightarrow \prod_{(u, s) \in X_{j}} \mathbf{R}_{s}$ so that $\bar{\rho}_{j}(a)(u, s)=\bar{\rho}_{u, s}(a)$ for $a \in S_{j}$. Let $\mathbf{S}^{(j)}$ and $\mathbf{S}_{0}^{(j)}$ be the images under $\bar{\rho}_{j} \pi_{j}^{S}$ of $\mathbf{S}$ and $\mathbf{S}_{0}$ respectively.

Choose $h \in \operatorname{Hom}(\mathbf{S}, \underline{\mathbf{R}})$ such that $\left.h\right|_{S_{0}}=h_{0}$. Fix $i \in\{1, \ldots, k\}$. As $\mathbf{R}_{i}$ is local and $\pi_{i}^{R} h \in \operatorname{Hom}\left(\mathbf{S}, \mathbf{R}_{i}\right)$, there exists a unique $j \in\{1, \ldots, \ell\}$ such that $\operatorname{ker}\left(\pi_{j}^{S}\right) \subseteq \operatorname{ker}\left(\pi_{i}^{R} h\right)$. Define $h^{i} \in \operatorname{Hom}\left(\mathbf{S}^{(j)}, \mathbf{R}_{i}\right)$ so that $h^{i} \bar{\rho}_{j} \pi_{j}^{S}=\pi_{i}^{R} h$, and let $h_{0}^{i}=\left.h^{i}\right|_{S_{0}^{(j)}}$. We have $\mathbf{S}_{0}^{(j)} \leq \mathbf{S}^{(j)} \leq \prod_{(u, s) \in X_{j}} \mathbf{R}_{s}, h_{0}^{i} \in \operatorname{Hom}\left(\mathbf{S}_{0}^{(j)}, \mathbf{R}_{i}\right)$, where $\mathbf{R}_{i}$ and $\mathbf{S}^{(j)}$ are local, and $h_{0}^{i}$ lifts to $\mathbf{S}^{(j)}$. Thus by Corollary 5.9 there exists $Y_{i} \subseteq X_{j}$ such that $h_{0}^{i}$ lifts to $\left.\mathbf{S}^{(j)}\right|_{Y_{i}}$ and $\left|Y_{i}\right| \leq\left|S_{0}^{(j)}\right| \cdot\left|R_{i} / J\left(\mathbf{R}_{i}\right)\right|$. Fix such $Y_{i}$ for each $i=1, \ldots, k$.

Let $Y=\left\{u \in U:(u, s) \in Y_{1} \cup \cdots \cup Y_{k}\right.$ for some $\left.s\right\}$. Then $|Y| \leq\left|S_{0}\right| \cdot|R / J(\underline{\mathbf{R}})|$ and the reader is invited to check that $h_{0}$ lifts to $\left.\mathbf{S}\right|_{Y}$.

\section{COROLLARY 7.3. TC( $\underline{\mathbf{R}})$ holds.}

Proof. By the previous claim and Theorem 4.11.

Now we turn to proving GenClon $(\Sigma, \underline{\mathbf{R}})$. Let $\mathbf{S}$ be an arbitrary subring of $\underline{\mathbf{R}}^{n}(n \geq 1)$ and define

$T=\left\{a \in R^{n}: \exists b \in S\right.$ such that $a_{i} \equiv b_{i} \bmod J(\underline{\mathbf{R}})$ for $\left.i=1, \ldots, n\right\}$.

Note that $\mathbf{S} \leq \mathbf{T} \leq \underline{\mathbf{R}}^{n}, \mathbf{S} / J(\mathbf{S}) \cong \mathbf{T} / J(\mathbf{T})$, and $J(\mathbf{T})=J(\underline{\mathbf{R}}) \times \cdots \times J(\underline{\mathbf{R}})$.

CLAIM 7.4. Every $h \in \operatorname{Hom}(\mathbf{S}, \underline{\mathbf{R}})$ can be extended to some $h^{+} \in \operatorname{Hom}(\mathbf{T}, \underline{\mathbf{R}})$. 
Proof. As $\mathbf{S}$ and $\mathbf{T}$ have the same idempotent elements, we can decompose $\mathbf{S} \cong \mathbf{S}_{1}$ $\times \cdots \times \mathbf{S}_{\ell}$ and $\mathbf{T} \cong \mathbf{T}_{1} \times \cdots \times \mathbf{T}_{\ell}$ and choose projections $\pi_{j}^{S}$ and $\pi_{j}^{T}$ as in the proof of Theorem 6.1 so that $\mathbf{S}_{j} \leq \mathbf{T}_{j}$ and $\pi_{j}^{S}=\left.\pi_{j}^{T}\right|_{S}$ for all $j=1, \ldots, \ell$.

Suppose $h \in \operatorname{Hom}(\mathbf{S}, \underline{\mathbf{R}})$, fix $i=1, \ldots, k$ and let $h_{i}=\pi_{i}^{R} h \in \operatorname{Hom}\left(\mathbf{S}, \mathbf{R}_{i}\right)$ and $p=\operatorname{char}^{*}\left(\mathbf{R}_{i}\right)$. It suffices to prove the existence of $h_{i}^{+} \in \operatorname{Hom}\left(\mathbf{T}, \mathbf{R}_{i}\right)$ such that $\left.h_{i}^{+}\right|_{s}=$ $h_{i}$. As $\mathbf{R}_{i}$ is local there exists a unique $j=1, \ldots, \ell$ such that $\operatorname{ker}\left(\pi_{j}^{S}\right) \subseteq \operatorname{ker}\left(h_{i}\right)$. Choose $\theta \in \operatorname{Hom}\left(\mathbf{S}_{j} / J\left(\mathbf{S}_{j}\right), \mathbf{R}_{i} / J\left(\mathbf{R}_{i}\right)\right)$ and $\bar{h}_{i} \in \operatorname{Hom}_{\theta}\left(\mathbf{S}_{j}, \mathbf{R}_{i}\right)$ so that $\bar{h}_{i} \pi_{j}^{S}=h_{i}$. Let $v: \mathbf{T}_{j} / J\left(\mathbf{T}_{j}\right) \cong \mathbf{S}_{j} / J\left(\mathbf{S}_{j}\right)$ be the isomorphism induced by the inclusion by $\mathbf{T}_{j}$ of $\mathbf{S}_{j}$, and let $\theta^{*}=v \theta$. As $\operatorname{Hom}_{\mathbf{p}}\left(J\left(\mathbf{S}_{j}\right), J_{\theta}\left(\mathbf{R}_{i}\right)\right)=\operatorname{Hom}_{\mathbf{p}}\left(J_{v}\left(\mathbf{S}_{j}\right), J_{\theta^{*}}\left(\mathbf{R}_{i}\right)\right)$ and because $J_{v}\left(\mathbf{S}_{j}\right)$ is a subspace of $J\left(\mathbf{T}_{j}\right)$, every $\lambda \in \operatorname{Hom}_{\mathbf{p}}\left(J\left(\mathbf{S}_{j}\right), J_{\theta}\left(\mathbf{R}_{i}\right)\right)$ can be extended to some $\lambda^{+} \in \operatorname{Hom}_{\mathbf{p}}\left(J\left(\mathbf{T}_{j}\right), J_{\theta^{*}}\left(\mathbf{R}_{i}\right)\right)$. It follows from Theorem 5.6 that $\bar{h}_{i}$ can be extended to some $\bar{h}_{i}^{+} \in \operatorname{Hom}\left(\mathbf{T}_{j}, \mathbf{R}_{i}\right)$. Now put $h_{i}^{+}=\bar{h}_{i}^{+} \pi_{j}^{T}$.

CLAIM 7.5. GenClon $(\Sigma, \underline{\mathbf{R}})$ holds. That is, if $\mathbf{S} \leq \underline{\mathbf{R}}^{n}$ and $h \in \operatorname{Hom}(\mathbf{S}, \underline{\mathbf{R}})$, then there exists an operation $h^{\prime}$ in the partial clone generated by $\Sigma$ so that $\mathbf{S} \leq \operatorname{dom}\left(h^{\prime}\right) \leq \underline{\mathbf{R}}^{n}$ and $\left.h^{\prime}\right|_{S}=h$.

Proof. Let $\operatorname{Clo}(\Sigma)$ denote the partial clone generated by $\Sigma$ (see Definition 4.5(1)). Let $\mathbf{S}$ be given. By the previous claim we may assume with no loss of generality that $J(\mathbf{S})=$ $J(\underline{\mathbf{R}}) \times \cdots \times J(\underline{\mathbf{R}})$. Choose $\mathbf{S}_{1}, \ldots, \mathbf{S}_{\ell}$ and $\pi_{1}^{S}, \ldots, \pi_{\ell}^{S}$ as usual. Let $h \in \operatorname{Hom}(\mathbf{S}, \underline{\mathbf{R}})$ be given and put $h_{i}=\pi_{i}^{R} h \in \operatorname{Hom}\left(\mathbf{S}, \mathbf{R}_{i}\right)$ for $i=1, \ldots, k$.

We shall show the following: for each $i=1, \ldots, k$ there exists $h_{i}^{\prime} \in \operatorname{Clo}(\Sigma)$ such that $\mathbf{S} \leq \operatorname{dom}\left(h_{i}^{\prime}\right) \leq \underline{\mathbf{R}}^{n}$ and $\left.\pi_{i}^{R} h_{i}^{\prime}\right|_{S}=h_{i}$. This will suffice, for then $h^{\prime}=d\left(h_{1}^{\prime}, \ldots, h_{k}^{\prime}\right)$ will have the desired properties.

So fix $i=1, \ldots, k$. Put $U=\{1, \ldots, n\}$ so that $\mathbf{S} \leq \underline{\mathbf{R}}^{U}$. As in the proof of Claim 7.2, choose $j$ so that $\operatorname{ker}\left(\pi_{j}^{S}\right) \subseteq \operatorname{ker}\left(h_{i}\right)$ and define $X_{j} \subseteq U \times\{1, \ldots, k\}$, define $\bar{\rho}_{j}: \mathbf{S}_{j} \cong$ $\mathbf{S}^{(j)} \leq \prod_{(u, s) \in X_{j}} \mathbf{R}_{s}$ and define $h^{i} \in \operatorname{Hom}\left(\mathbf{S}^{(j)}, \mathbf{R}_{i}\right)$ so that $h^{i} \bar{\rho}_{j} \pi_{j}^{S}=h_{i}$. Note that $J\left(\mathbf{S}^{(j)}\right)=\prod_{(u, s) \in X_{j}} J\left(\mathbf{R}_{s}\right)$ by the assumption on $\mathbf{S}$.

Choose $\theta \in \operatorname{Hom}\left(\mathbf{S}^{(j)} / J\left(\mathbf{S}^{(j)}\right), \mathbf{R}_{i} / J\left(\mathbf{R}_{i}\right)\right)$ so that $h^{i} \in \operatorname{Hom}_{\theta}\left(\mathbf{S}^{(j)}, \mathbf{R}_{i}\right)$ and define

$$
\begin{aligned}
\mathcal{C}= & \left\{f \in \operatorname{Hom}_{\theta}\left(\mathbf{S}^{(j)}, \mathbf{R}_{i}\right): \exists f^{\prime} \in \operatorname{Clo}(\Sigma)\right. \text { such that } \\
& \left.\mathbf{S} \leq \operatorname{dom}\left(f^{\prime}\right) \leq \underline{\mathbf{R}}^{n} \text { and }\left.\pi_{i}^{R} f^{\prime}\right|_{S}=f \bar{\rho}_{j} \pi_{j}^{S}\right\} .
\end{aligned}
$$

It suffices to show $\mathcal{C}=\operatorname{Hom}_{\theta}\left(\mathbf{S}^{(j)}, \mathbf{R}_{i}\right)$. The idea is to

1. Show that $\mathcal{C}$ contains every $f \in \operatorname{Hom}_{\theta}\left(\mathbf{S}^{(j)}, \mathbf{R}_{i}\right)$ whose restriction to $J\left(\mathbf{S}^{(j)}\right)$ depends on at most two coordinates (in $X_{j}$ ), and

2. Show that $\mathcal{C}$ is closed under $x-y+z$, and then

3. Repeat the argument of Cases 1 and 2 in the proof of Theorem 6.1. 
To prove the first item, suppose $f \in \operatorname{Hom}_{\theta}\left(\mathbf{S}^{(j)}, \mathbf{R}_{i}\right), \lambda=\left.f\right|_{J\left(\mathbf{S}^{(j)}\right)}$, and $\lambda$ depends only on the coordinates $\left(u_{1}, s_{1}\right),\left(u_{2}, s_{2}\right) \in X_{j}$. Thus for all $a \in J\left(\mathbf{S}^{(j)}\right)$, if $a_{\left(u_{t}, s_{t}\right)}=0$ for $t=1,2$ then $\lambda(a)=0$. We claim that for all $a \in S^{(j)}$, if $a_{\left(u_{t}, s_{t}\right)}=0$ for $t=1,2$ then $f(a)=0$. This follows from the fact that if $a \in S^{(j)}$ and $a_{(u, s)} \in J\left(\mathbf{R}_{s}\right)$ for some $(u, s) \in X_{j}$, then $a_{(u, s)} \in J\left(\mathbf{R}_{s}\right)$ for all $(u, s) \in X_{j}$, as $\mathbf{S}^{(j)}$ is local. Hence $f$ depends only on the coordinates $\left(u_{1}, s_{1}\right),\left(u_{2}, s_{2}\right)$.

Define $\mathbf{T}_{0} \leq \underline{\mathbf{R}}^{2}, \mathbf{T} \leq \underline{\mathbf{R}}^{n}, g_{0} \in \operatorname{Hom}\left(\mathbf{T}_{0}, \underline{\mathbf{R}}\right)$, and $g \in \operatorname{Hom}(\mathbf{T}, \underline{\mathbf{R}})$ as follows:

$$
\begin{aligned}
& T_{0}=\left\{\left(a_{s_{1}}, a_{s_{2}}\right): a \in S\right\} \\
& T=\left\{b \in R^{n}:\left(b_{s_{1}}, b_{s_{2}}\right) \in ـ_{-} 0\right\} \\
& \pi_{t}^{R} g_{0}\left(a_{s_{1}}, a_{s_{2}}\right)=\left\{\begin{array}{ll}
f \bar{\rho}_{j} \pi_{j}^{S}(a) & \text { if } t=i \\
\pi_{t}^{R}\left(a_{s_{1}}\right) & \text { if } t \neq i
\end{array}, a \in S\right. \\
& g(b)=g_{0}\left(b_{s_{1}}, b_{s_{2}}\right), \quad b \in T \text {. }
\end{aligned}
$$

We have $g_{0} \in \mathcal{F}_{2} \subseteq \Sigma$ and hence $g \in \operatorname{Clo}(\Sigma)$. As $\mathbf{S} \leq \mathbf{T}$ and $\left.\pi_{i}^{R} g\right|_{S}=f \bar{\rho}_{j} \pi_{j}^{S}$, we get $f \in \mathcal{C}$ as desired.

The proof of the second item is left to the reader. Now let $f \in \operatorname{Hom}_{\theta}\left(\mathbf{S}^{(j)}, \mathbf{R}_{i}\right)$ be arbitrary; put $p=\operatorname{char}^{*}\left(\mathbf{R}_{i}\right)$ and argue by cases.

CASE 1. $\mathbf{p}_{S^{(j)}}=0$.

Let $\left\{e_{1}, \ldots, e_{m}\right\}$ be a basis for $J\left(\mathbf{S}^{(j)}\right)$. Since $J\left(\mathbf{S}^{(j)}\right)=\prod_{(u, s) \in X_{j}} J\left(\mathbf{R}_{s}\right)$, the basis can be chosen so that each $e_{t}$ is zero at every coordinate in $X_{j}$ except one. Now define $\lambda_{t}$ and $g_{t}(t=0, \ldots, m)$ as in the proof of Case 1 in Section 6 but with $f$ in place of $h$. We have $g_{t} \in \operatorname{Hom}_{\theta}\left(\mathbf{S}^{(j)}, \mathbf{R}_{i}\right)$ for $t=1, \ldots, m$, each $\left.g_{t}\right|_{J\left(\mathbf{S}^{(j)}\right)}$ depends on at most one coordinate in $X_{j}$, and $f$ is in the closure (in $\operatorname{Hom}_{\theta}\left(\mathbf{S}^{(j)}, \mathbf{R}_{i}\right)$ ) of $\left\{g_{0}, \ldots, g_{m}\right\}$ under $x-y+z$. Thus $f \in \mathcal{C}$.

\section{CASE 2. $\mathbf{p}_{S^{(j)}} \neq 0$.}

As in Section 6, let $\left\{e_{0}, \ldots, e_{m}\right\}$ be a basis for $J\left(\mathbf{S}^{(j)}\right)$ such that $\sum_{t} e_{t}=\mathbf{p}_{S^{(j)}}$. This basis may be chosen so that each $e_{t}$ except possibly $e_{0}$ is zero at every coordinate in $X_{j}$ except one. Define $\lambda_{t}$ and $g_{t}(t=0, \ldots, m)$ as in the proof of Case 2 in Section 6 but with $f$ in place of $h$. This time, each $g_{t}$ depends on at most two coordinates in $X_{j}$. The rest of the proof is the same.

And this completes the proof of Theorem 7.1.

In closing, note that if for each $i=1, \ldots, k$ we define $d_{i}: \underline{\mathbf{R}}^{2} \rightarrow \underline{\mathbf{R}}$ so that for all $a, b \in R, \pi_{i}^{R} d_{i}(a, b)=\pi_{i}^{R}(a)$ while $\pi_{j}^{R} d_{i}(a, b)=\pi_{j}^{R}(b)$ for $j \neq i$, then $\{d\}$ and 
$\left\{d_{1}, \ldots, d_{k}\right\}$ generate the same partial clone. Thus $\mathbb{I S P}(\underline{\mathbf{R}})$ is also strongly dualized by $\Sigma_{0} \cup \mathcal{F}_{2} \cup\left\{d_{1}, \ldots, d_{k}\right\}$. This proves (3) $\Rightarrow$ (2) in Theorem 2.1:

COROLLARY 7.6. If $\underline{\mathbf{R}}$ is a finite commutative ring with identity such that $J(\underline{\mathbf{R}})^{2}=\{0\}$, then $\operatorname{ISP}(\underline{\mathbf{R}})$ is strongly dualized by the set of all algebraic (partial) operations and relations of $\underline{\mathbf{R}}$ of arity at most 3 .

\section{The ring $\mathbb{Z}_{p^{2}}$ of integers modulo $p^{2}$}

Let $\underline{\mathbf{R}}$ be a finite commutative ring with identity whose radical is self annihilating. In order to use Theorem 7.1 to obtain a useful strong duality for $\mathbb{I S P}(\underline{\mathbf{R}})$, it remains

1. to identify the algebraic binary partial operations and ternary relations that constitute the bulk of the set $\Sigma$ defined in Theorem 7.1,

2. to derive from $\Sigma$ a small subset $\Sigma^{\prime}$ that also strongly dualizes $\mathbb{I S P}(\underline{\mathbf{R}})$ and is simple enough to allow us

3. to find a useful description of the objects in the dual category $\mathcal{X}:=\mathbb{I S}_{c} \mathbb{P}^{+}(\mathbf{R})$ determined by $\Sigma^{\prime}$.

In this section we illustrate how these three tasks can be successfully carried out when $\underline{\mathbf{R}}=\mathbb{Z}_{p^{2}}$. The results appear in Corollary 8.4, Theorem 8.6 and Theorem 8.10.

We henceforth write $\mathbb{Z}_{p^{2}}$ as $\underline{\mathbf{Z}}_{p^{2}}$. We begin by identifying precisely the members of the dualizing set $\Sigma$ of Theorem 7.1 when $\underline{\mathbf{R}}=\underline{\mathbf{Z}}_{p^{2}}$. We denote the radical of $\underline{\mathbf{Z}}_{p^{2}}$ by $J:=\{0, p, 2 p, \ldots,(p-1) p\}$.

CLAIM 8.1. The ring $\underline{\mathbf{Z}}_{p^{2}} \times \underline{\mathbf{Z}}_{p^{2}}$ has exactly three subrings: itself, the diagonal subring $\Delta=\left\{(a, a): a \in \mathbb{Z}_{p^{2}}\right\}$, and the subring $\equiv_{J}=\{(a, b): a-b \in J\}$.

For each $i=0,1, \ldots, p-1$, define the binary partial operation $g_{i}: \equiv_{J} \rightarrow \underline{\mathbf{Z}}_{p^{2}}$ by $g_{i}(a, b)=i a+(1-i) b$. Each $g_{i}$ is a ring homomorphism. Note that $g_{0}$ and $g_{1}$ are the two projections on $\equiv_{J}$.

CLAIM 8.2. If $h$ is a homomorphism from a subring of $\underline{\mathbf{Z}}_{p^{2}} \times \underline{\mathbf{Z}}_{p^{2}}$ to $\underline{\mathbf{Z}}_{p^{2}}$, then either $h$ is the first or second projection on its domain, or the domain of $h$ is $\equiv_{J}$ and $h=g_{i}$ for some $i<p$.

We next determine the subrings of $\left(\underline{\mathbf{Z}}_{p^{2}}\right)^{3}$. We continue to write $\equiv_{J}$ to denote the relation of equivalence modulo $J$. Recall that one subring of $\left(\underline{\mathbf{Z}}_{p^{2}}\right)^{3}$ is given by the set $D=\left\{(a, b, c) \in \mathbb{Z}_{p^{2}}^{3}: a \equiv_{J} b \equiv_{J} c\right\}$. 
CLAIM 8.3. The subrings of $\left(\underline{\mathbf{Z}}_{p^{2}}\right)^{3}$ are $\left(\underline{\mathbf{Z}}_{p^{2}}\right)^{3}$, D, the graphs of homomorphisms from subrings of $\underline{\mathbf{Z}}_{p^{2}} \times \underline{\mathbf{Z}}_{p^{2}}$ to $\underline{\mathbf{Z}}_{p^{2}}$ possibly followed by a permutation of the three coordinates, and $\mathbf{B}_{12}, \mathbf{B}_{13}$ and $\mathbf{B}_{23}$ where

$$
\begin{aligned}
& B_{12}:=\left\{(a, b, c) \mid a \equiv_{J} b\right\}, \\
& B_{13}:=\left\{(a, b, c) \mid a \equiv_{J} c\right\}, \\
& B_{23}:=\left\{(a, b, c) \mid b \equiv_{J} c\right\} .
\end{aligned}
$$

Together, Claims 8.1, 8.2 and 8.3 give us an exact description of the set $\Sigma$ defined in Theorem 7.1 when $\underline{\mathbf{R}}$ is $\underline{\mathbf{Z}}_{p^{2}}$.

COROLLARY 8.4. IISP $\left(\underline{\mathbf{Z}}_{p^{2}}\right)$ is strongly dualized by the set $\Sigma$ consisting of

1. the partial ternary operation $x-y+z$ restricted to $D$,

2. the two projections on each of $\mathbb{Z}_{p^{2}} \times \mathbb{Z}_{p^{2}}, \Delta$ and $\equiv_{J}$ and the operations $g_{i}, 2 \leq i<p$;

3. the ternary relations $\left(\mathbb{Z}_{p^{2}}\right)^{3}, D, B_{12}, B_{13}, B_{23}$, and the graphs of the partial binary operations in item 2 under all possible permutations of the coordinates;

4. the identity map $\mathbb{Z}_{p^{2}} \rightarrow \mathbb{Z}_{p^{2}}$.

The next step is to extract from $\Sigma$ a small subset $\Sigma^{\prime}$ which still strongly dualizes $\mathbb{I S P}(\underline{\mathbf{Z}})$. General algorithms to do this are a major theme of the text [2]; see Section 2.4 and Chapters 8 and 9 . We are fortunate that, in the present case, the reduction can be achieved easily from first principles.

LEMMA 8.5. Assume $\underline{\mathbf{M}}$ is a finite algebra, $\Sigma$ and $\Sigma^{\prime}$ are sets of finitary operations and relations algebraic over $\underline{\mathbf{M}}$, and $\mathbb{I S P}(\underline{\mathbf{M}})$ is strongly dualized by $\Sigma$. Then $\mathbb{I S P}(\underline{\mathbf{M}})$ is also strongly dualized by $\Sigma^{\prime}$ provided that

1. for every set $S$, every subset $X \subseteq M^{S}$, and every map $\varphi: X \rightarrow M$, if $\varphi$ preserves the members of $\Sigma^{\prime}$, then $\varphi$ also preserves the members of $\Sigma$;

2. each partial operation of $\Sigma$ is in the partial clone generated by $\Sigma^{\prime}$.

Proof. To see that $\operatorname{ISP}(\underline{\mathbf{M}})$ is dualized by $\Sigma^{\prime}$, suppose $\mathbf{A} \in \mathbb{I S P}(\underline{\mathbf{M}})$ and let $\varphi$ : $\operatorname{Hom}(\mathbf{A}, \underline{\mathbf{M}}) \rightarrow M$ be continuous and preserve each member of $\Sigma^{\prime}$. Then $\varphi$ also preserves each member of $\Sigma$, by item 1 , and therefore is $e^{\mathbf{A}}(a)$ for some $a \in A$. Thus $e^{\mathbf{A}}$ is surjective with respect to $\Sigma^{\prime}$.

By Lemma 4.6, $\operatorname{TC}(\Sigma, \underline{\mathbf{M}})$ holds and it suffices to prove that $\operatorname{TC}\left(\Sigma^{\prime}, \underline{\mathbf{M}}\right)$ holds. A topologically closed substructure of a power of $\left\langle M ; \Sigma^{\prime}\right\rangle$ is, by item 2 , also a topologically closed substructure of a power of $\langle M ; \Sigma\rangle$ and is therefore term-closed by $\operatorname{TC}(\Sigma, \underline{\mathbf{M}})$. This proves $\operatorname{TC}\left(\Sigma^{\prime}, \underline{\mathbf{M}}\right)$.

THEOREM 8.6. $\mathbb{I S P}\left(\underline{\mathbf{Z}}_{p^{2}}\right)$ is strongly dualized by the set $\Sigma^{\prime}=\left\{x-y+\left.z\right|_{D}, \equiv_{J}\right\}$. 
Proof. We take $\Sigma$ as given in Corollary 8.4 and verify the conditions of Lemma 8.5. Assume that $\varphi: X \rightarrow M$ preserves the members of $\Sigma^{\prime}$. Trivially every map $X \rightarrow M$ preserves the identity map $\mathbb{Z}_{p^{2}} \rightarrow \mathbb{Z}_{p^{2}}$ as well as the projections on $\mathbb{Z}_{p^{2}} \times \mathbb{Z}_{p^{2}}$ and on $\Delta$, and preserves the set $\mathbb{Z}_{p^{2}} \times \mathbb{Z}_{p^{2}}$ itself. Since $\varphi$ preserves $\equiv_{J}$, it preserves both of its projections as well as $B_{12}, B_{13}$ and $B_{23}$. If $\varphi$ preserves a partial operation, then it trivially preserves the graph of that partial operation under all permutations of coordinates, as well as any other operation in the partial clone it generates. It therefore remains only to verify that each partial operation $g_{i}$ is in the partial clone generated by $x-y+\left.z\right|_{D}$. This follows from the fact that $g_{0}$ and $g_{1}$ are projections, and from the recursion $g_{i+1}(a, b)=$ $g_{i}(a, b)-b+a$.

The final step is to give a description of the dual category $\mathcal{X}=\mathbb{I} \mathbb{S}_{c} \mathbb{P}^{+}\left(\mathbf{Z}_{p^{2}}\right)$ where

$$
\mathbf{Z}_{p^{2}}:=\left\langle\mathbb{Z}_{p^{2}} ; x-y+\left.z\right|_{D}, \equiv_{J}, \text { discrete topology }\right\rangle
$$

is the topological structure determined by $\Sigma^{\prime}$. Relevant properties of such dual categories are presented in Section 1.4 of Clark and Davey [2]. We will extract the necessary information from [2] by quoting the following two theorems, where we have replaced the structure $\underset{\sim}{\mathbf{M}}$ of arbitrary type by the particular structure $\underset{\sim}{\mathbf{Z}_{p^{2}}}$.

LEMMA 8.7. ([2], Theorem 1.4.3.) If $\mathbf{X}=\langle X ; f, \equiv, \mathcal{T}\rangle \in \mathbb{I} \mathbb{S}_{c} \mathbb{P}^{+}\left(\mathbf{Z}_{p^{2}}\right)$, then

1. $\mathbf{X}$ is a structured Boolean space which satisfies every quasi-atomic formula (equation or implication) that is satisfied by $\mathbf{Z}_{p^{2}}$,

2. $f$ is a continuous partial ternary operation whose domain is a closed subset of $X^{3}$, and

3. $\equiv$ is a closed subset of $X^{2}$.

LEMMA 8.8. ([2], Theorem 1.4.4.) Let $\mathbf{X}=\langle X ; f, \equiv, \mathcal{T}\rangle$ be a Boolean space with a ternary partial operation $f$ and a binary relation $\equiv$. Then $\mathbf{X} \in \mathbb{I} \mathbb{S}_{c} \mathbb{P}^{+}\left(\mathbf{Z}_{p^{2}}\right)$ if and only if

1. for each $x, y \in X$ where $x \neq y$, there is a morphism $\alpha: \mathbf{X} \rightarrow{\underset{\sim}{p^{2}}}_{\text {such that }}$ $\alpha(x) \neq \alpha(y)$,

2. for each $(x, y, z) \in X^{3}$ not in dom $(f)$, there is an $\alpha: \mathbf{X} \rightarrow \mathbf{Z}_{p^{2}}$ such that $(\alpha(x), \alpha(y)$, $\alpha(z)) \notin D$, and

3. for each $x, y \in X$ where $x \not \equiv y$, there is an $\alpha: \mathbf{X} \rightarrow{\underset{\sim}{Z^{2}}}_{p^{2}}$ such that $\alpha(x) \not \equiv_{J} \alpha(y)$.

We first examine the topological quasivariety $\mathbb{I} \mathbb{S}_{c} \mathbb{P}^{+}(\mathbf{J})$ generated by the substructure $\underset{\sim}{\mathbf{J}}=\langle J ; f, \equiv, \mathcal{T}\rangle$ of $\mathbf{Z}_{p^{2}}$ where $f(x, y, z)=x-y+z$ is now a total ternary operation and $\equiv$ is the universal relation. An affine Boolean $p$-space is a structured Boolean space $\mathbf{X}=\langle X ; f, \equiv, \mathcal{T}\rangle$ which carries the universal relation $\equiv$ and has an underlying continuous binary operation + such that $\langle X,+\rangle$ is a topological vector space over $\mathbb{Z}_{p}$ and 
$f(x, y, z)=x-y+z$. The structure $\mathbf{J}$ is an example of an affine Boolean $p$-space. We use a result of Davey and Werner [9] to establish the following characterization of these structures.

LEMMA 8.9. $\mathbb{I S}_{c} \mathbb{P}^{+}(\underset{\sim}{\mathbf{J}})$ is the category of all affine Boolean p-spaces.

Proof. It is immediate to check that isomorphic copies and direct products of affine Boolean $p$-spaces are again affine Boolean $p$-spaces. Let $\langle Y ; f, \equiv, \mathcal{T}\rangle$ be a substructure of an affine Boolean $p$-space $\langle X ; f, \equiv, \mathcal{T}\rangle$. Then for any $a \in Y$, the operation $x+a$ $y=x-a+y$ makes $Y$ into an affine Boolean $p$-space, and it is easy to check that $f(x, y, z)=x-{ }_{a} y+{ }_{a} z$ for $x, y, z \in Y$. It follows from Lemma 8.7 that each member of $\mathbb{I} \mathbb{S}_{c} \mathbb{P}^{+}(\underset{J}{\mathbf{J}})$ is an affine Boolean $p$-space.

Conversely, we will use Lemma 8.8 to show that any affine Boolean $p$-space $\langle X ; f, \equiv, \mathcal{T}\rangle$ is in $\mathbb{S}_{\mathcal{C}} \mathbb{P}^{+}(\underset{\mathbf{J}}{X}$. Since $f$ is total and $\equiv$ is universal, it remains only to verify the first item. Let $r, s \in \mathcal{X}$ where $r \neq s$. By Davey and Werner [9], there is a continuous homomorphism $\varphi$ from the Boolean linear space $\langle X ;+, \mathcal{T}\rangle$ over $\mathbb{Z}_{p}$ into the discrete linear space $\langle J ;+, \mathcal{T}\rangle$ over $\mathbb{Z}_{p}$ which separates $r$ and $s$. (See also [2], 1.4.8.) As $\varphi$ also preserves the ternary operation $f(x, y, z)=x-y+z$, it provides the required continuous homomorphism.

THEOREM 8.10. A structure $\mathbf{X}=\langle X ; f, \equiv, \mathcal{T}\rangle$ is in $\mathbb{I S}_{c} \mathbb{P}^{+}\left(\underset{\sim}{\mathbf{Z}_{p^{2}}}\right)$ if and only if $\langle X, \mathcal{T}\rangle$ is a Boolean space, $f$ is a continuous ternary partial operation on $X$ and $\equiv$ is an equivalence relation on $X$ such that

1. the domain of $f$ is the union of all $T^{3}$ where $T$ ranges over the $\equiv$-classes;

2. each $\equiv$-class is a closed subset of $\mathbf{X}$ which forms an affine Boolean p-space under $f$;

3. if $x \neq \equiv$ in $\mathbf{X}$, then there is a partition of $\mathbf{X}$ into two disjoint clopen unions of $\equiv$-classes, one containing $x$ and the other containing $y$.

Proof. We apply Lemma 8.7 to check that each member $\mathbf{X}$ of $\mathbb{S _ { C }} \mathbb{P}^{+}\left({\underset{\sim}{p^{2}}}^{2}\right)$ has each of these properties by checking the quasi-equations which hold in $\underset{\sim}{\mathbf{Z}} p^{2}$. First, we observe that the defining properties of an equivalence relation are all quasi-equational. Item 1 is a consequence of the pair of quasi-equations

$$
f(x, y, z) \approx f(x, y, z) \longleftrightarrow x \equiv y \equiv z,
$$

where we recall $[2, \S 1.4]$ that satisfaction on an equation involving partial operations requires that both sides be defined. Each $\equiv$-class must be closed since $\equiv$ is closed in $X^{2}$. Item 2 now follows by writing the axioms of an affine $p$-space as a set of equations, which we can do since the underlying addition operation can be taken to be $x+{ }_{a} y=f(x, a, y)$ for

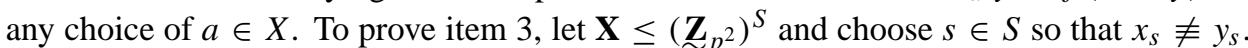
Define $\psi: \mathbf{X} \rightarrow \mathbf{Z}_{p^{2}}$ as $\psi(u)=0$ if $u_{s}=x_{s}$ and $\psi(u)=1$ if $u_{s} \neq x_{s}$. Then $\psi^{-1}(0)$ and $\psi^{-1}(1)$ provide the required partition of $\mathbf{X}$. 
Now suppose that $\mathbf{X}$ is a structure with the listed properties. We will verify the conditions of Lemma 8.8. Beginning with the third condition, assume $x, y \in X$ where $x \neq \equiv y$. Let $x \in U$ and $y \in V$ where $U$ and $V$ partition $X$ into two disjoint clopen unions of $\equiv$-classes. Then the map $\alpha$ taking $U$ to 0 and $V$ to 1 is the required morphism since $0 \not_{J} 1$. To prove the second condition, suppose $(x, y, z) \in X^{3} \backslash \operatorname{dom}(f)$. By item 1 , either $x \not \equiv y$ or $y \not \equiv z$, and the result follows from the third condition.

A bit more effort is required to verify the first condition. Choose $x, y \in X$ where $x \neq y$ and where, in view of the third condition, we may assume that $x \equiv y$. Let $T$ be the $\equiv$-class of $x$ and $y$. Since $\mathbf{T}$ forms an affine Boolean $p$-space, Lemma 8.9 tells us that there is a morphism $\varphi: \mathbf{T} \rightarrow \underset{\sim}{\mathbf{J}}$ that separates $x$ and $y$. Our goal is to show that $\varphi$ has an extention $\psi$ from $\mathbf{X}$ into $\underset{\sim}{\mathbf{Z}_{p^{2}}}$.

For $i=0,1, \ldots, p-1$, the inverse images $\varphi^{-1}(i)$ partition $T$ into disjoint subsets which are clopen in $T$. Let $U_{0}, U_{1}, \ldots, U_{p-1}$ be disjoint clopen subsets of $X$ such that $\varphi^{-1}(i) \subseteq U_{i}$, and let $U$ be their union.

CLAIM 8.11. There is a clopen union $V$ of $\equiv$-classes containing $T$ and contained in $U$.

Proof. For each point $r$ in $X \backslash U$, we can apply item 3 to obtain a clopen union of $\equiv$-classes which contains $r$ and does not intersect $T$. As $X \backslash U$ is compact, finitely many of these sets cover $X \backslash U$. We take $V$ to be the complement of the union of these finitely many.

We now form a clopen partition of $V$ by defining $V_{i}:=V \cap U_{i}$ for $i=0,1, \ldots, p-1$. Items 2 and 3 tell us that $\operatorname{dom}(f)$ is a closed subset of $X^{3}$, and therefore its intersection $D_{V}$ with $V^{3}$ is a closed subset of $V^{3}$. As $f: D_{V} \rightarrow V$ is continuous, the inverse images $f^{-1}\left(V_{i}\right)$, where $i=0,1, \ldots, p-1$, form a clopen partition of $D_{V}$ in the relative topology. Each point of $f^{-1}\left(V_{i}\right)$ is therefore contained in a basic clopen subset $A \times B \times C$ of $V^{3}$ where $A, B, C \subseteq V$ are clopen and $A \times B \times C$ does not intersect $f^{-1}\left(V_{j}\right)$ if $j \neq i$. As $D_{V}$ is compact, only finitely many sets $A \times B \times C$ are required.

Our next step is to close the finite collection of clopen sets $A$ (which cover $V$ ) under intersection and complement, and let $\mathcal{A}$ be the minimal members of this new collection. Then $\mathcal{A}$ is a finite clopen partition of $V$. Similarly, construct clopen partitions $\mathcal{B}$ and $\mathcal{C}$ of $V$ so that $A \in \mathcal{A}, B \in \mathcal{B}$ and $C \in \mathcal{C}$ implies that $A \times B \times C$ intersects only one $f^{-1}\left(V_{i}\right)$.

Let $\mathcal{D}$ be a finite common clopen refinement of the partitions $\mathcal{A}, \mathcal{B}$ and $\mathcal{C}$ of $V$. Thus each $D \in \mathcal{D}$ is contained in a member of $\mathcal{A}$, a member of $\mathcal{B}$ and a member of $\mathcal{C}$. This means that $\mathcal{D}$ is a clopen partition of $V$ such that $D, E, F \in \mathcal{D}$ implies that $D \times E \times F$ intersects at most one $f^{-1}\left(V_{i}\right)$.

Let $\mathcal{E}=\{D \in \mathcal{D} \mid D \cap T \neq \varnothing\}$. As proven in Claim 8.11, we can find a clopen union $W$ of $\equiv$-classes containing $T$ which is contained in $\bigcup \mathcal{E}$. The intersections of the members of $\mathcal{E}$ with $W$ form a partition $\mathcal{F}$ of $W$ into clopen sets such that

1. $F \in \mathcal{F}$ implies that $F \cap T \neq \varnothing$ and

2. if $F, G, H \in \mathcal{F}$, then for some $i=0,1, \ldots, p-1$ the intersection $(F \times G \times H) \cap$ $\operatorname{dom}(f)$ is contained in $f^{-1}\left(V_{i}\right)$. 
CLAIM 8.12. Each member of $\mathcal{F}$ is contained in some (unique) $V_{i}$.

Proof. Let $r, s \in F \in \mathcal{F}$. Taking $G=H=F$ in 2 above, we find a $V_{i}$ such that $F^{3} \cap \operatorname{dom}(f) \subseteq f^{-1}\left(V_{i}\right)$. Thus $(r, r, r),(s, s, s) \in f^{-1}\left(V_{i}\right)$ and we have $r=f(r, r, r) \in$ $V_{i}$ and $s=f(s, s, s) \in V_{i}$ are in the same member of the clopen partition of $V$.

CLAIM 8.13. The map $\psi: \mathbf{W} \rightarrow \underset{\sim}{\mathbf{J}}$, taking $x$ to $i$ if $x \in V_{i}$, is a continuous morphism which extends $\varphi$.

Proof. Since each $V_{i}$ is clopen, $\psi$ is continuous, and $\psi$ trivially preserves $\equiv$ and extends $\varphi$ since $\varphi^{-1}(i) \subseteq V \cap U_{i}=V_{i}$. To see that it preserves $f$, choose $(u, v, w) \in W^{3} \cap \operatorname{dom}(f)$. Let $F, G, H \in \mathcal{F}$ and $r, s, t \in T$ such that $u, r \in F$, that $v, s \in G$ and that $w, t \in H$. Choose $i$ so that $(F \times G \times H) \cap \operatorname{dom}(f) \subseteq f^{-1}\left(V_{i}\right)$. Then $f(u, v, w), f(r, s, t) \in V_{i}$. By Claim 8.12, $\psi$ is constant on each member of $\mathcal{F}$. Then

$$
\begin{aligned}
\psi(f(u, v, w)) & =i=\psi(f(r, s, t))=\varphi(f(r, s, t))=f(\varphi(r), \varphi(s), \varphi(t)) \\
& =f(\psi(r), \psi(s), \psi(t))=f(\psi(u), \psi(v), \psi(w)) .
\end{aligned}
$$

To complete the verification of item 1 in Theorem 8.10, recall that $\mathbf{T} \subseteq \mathbf{W}$ and that $\varphi$ : $\mathbf{T} \rightarrow \mathbf{J}$ separates $x$ and $y$. Since $W$ is a clopen union of $\equiv$-classes, its complement is as well. We now extend $\psi$ to a continuous morphism from $\mathbf{X}$ to ${\underset{\sim}{p^{2}}}_{p^{2}}$ by taking all of $X \backslash W$ to any single element of $\underset{\sim}{\mathbf{Z}} p^{2}$.

\section{REFERENCES}

[1] ARens, R. F. and KAPlansky, I., Topological representation of algebras. Trans. Amer. Math. Soc. 63 (1968), 457-481.

[2] Clark, D. M. and Davey, B. A., Natural Dualities for the Working Algebraist. Cambridge University Press, 1998.

[3] Clark, D. M. and Davey, B. A., The quest for strong dualities. J. Austral. Math. Soc. (Series A), 58 (1995), 248-280.

[4] Clark, D. M., Davey, B. A. and Pitkethly, J. G., Dualisability of three element unary algebras. Manuscript, 2000.

[5] Clark, D. M. and Krauss, P. H., Topological quasi-varieties. Acta Sci. Math. 47 (1984), 3-39.

[6] Davey, B. A., Dualisability in general and endodualisability in particular. In: A. Ursini and P. Aglianò, editors, Logic and Algebra (Pontignano, 1994), volume 180 of Lecture Notes in Pure and Applied Mathematics, pages 437-455. Marcel Dekker, New York, 1996.

[7] Davey, B. A., HaVIAR, M. and PRIESTLEY, H., The syntax and semantics of entailment in duality theory J. Symb. Logic 60 (1995), 1087-1114.

[8] DAVEy, B.A., HeINDORF, L. and MCKenZIE, R., Near unanimity: an obstacle to general duality theory Algebra Universalis 33 (1995), 428-439.

[9] Davey, B. A. and Werner, H., Dualities and equivalences for varieties of algebras. In A. P. Huhn and E. T. Schmidt, editors, Contributions to Lattice Theory (Szeged, 1980), volume 33 of Colloq. Math. Soc. János Bolyai, pages 101-275. North-Holland, 1983.

[10] Davey, B. A. and Willard, R., The dualisability of a quasi-variety is independent of the generating algebra. Algebra Universalis 45 (2001), 103-106. 
[11] DzIOBIAK, W., An answer on some three questions. Manuscript, 1989.

[12] GRÄTZER, G., Universal Algebra. D. van Nostrand Co., 1968.

[13] Hyndman, H., Mono-unary algebras are strongly dualizable. J. Austral. Math. Soc., Series A (to appear).

[14] LAMBEK, J., Lectures on Rings and Modules. Blaisdell Publishing Co., 1966.

[15] Lampe, W. A., McNulty, G. F. and Willard, R., Full duality among graph algebras and flat graph algebras. Algebra Universalis 45 (2001), 311-334.

[16] Quackenbush, R. and Szabó, Cs., Finite nilpotent groups are not dualizable. J. Austral. Math. Soc., Series A (to appear).

[17] SARAmago, M., A study of natural dualities, including an analysis of the structure of failsets. PhD Thesis: Universidade de Lisboa, 1998.

[18] Stone, M. H., The theory of representations for boolean algebras. Trans. Amer. Math. Soc. 4 (1936), 37-111.

[19] WILlARD, R., New tools for proving dualizability. In: Dualities, Interpretability and Ordered Structures, J. Vaz de Carvalho and I. Ferrèirim (eds), C.A.U.L., Lisbon, 1999, 69-74.

[20] ZÁdORI, L., Natural duality via a finite set of relations. Bull. Austral. Math. Soc. 51 (1995), 469-478.

\section{M. Clark}

Department of Mathematics

SUNY at New Paltz

New Paltz

NY 12561

USA

e-mail: clarksbox@hotmail.com

\section{P. M. Idziak}

Computer Science Department

Jagiellonian University

Kraków

Poland

e-mail:idziak@ii.uj.edu.pl

L. R. Sabourin

Department of Mathematics and Statistics

Queen's University

Kingston

Ontario K7L $3 N 6$

Canada

e-mail: sabourin@mast.queensu.ca

C. Szabó

Department of Algebra and Number Theory

Eötvös Loránd University

Budapest

Hungary

e-mail:csaba@cs.elte.hu

R. Willard

Department of Pure Mathematics

University of Waterloo

Waterloo

Ontario N2L $3 G 1$

Canada

e-mail:rdwillar@uwaterloo.ca 